\title{
Robust Integrated Design of Processes with Terminal Penalty Model Predictive Controllers
}

\author{
M. Francisco ${ }^{\mathrm{a} *}$, P. Vega ${ }^{\mathrm{a}}$, H. Álvarez ${ }^{\mathrm{b}}$ \\ ${ }^{a}$ University of Salamanca, Computing and Automation Department \\ Escuela Técnica Superior de Ingeniería Industrial. Av. Fernando Ballesteros s/n 37700 Béjar (Salamanca, Spain) \\ e-mail: mfs@usal.es; pvega@usal.es \\ ${ }^{b}$ Universidad Nacional de Colombia , Sede Medellín (Colombia). \\ Escuela de Procesos y Energía. Facultad de Minas.e-mail: hdalvare@unal.edu.co
}

Corresponding author: Tel: +34923294500 ext. 1309. Fax: + 34 923294514. E-mail address: mfs@usal.es (M. Francisco)

\begin{abstract}
In this work, a novel methodology for the Integrated Design (ID) of processes with linear Model Predictive Control (MPC) is addressed, providing simultaneously the plant dimensions, the control system parameters and a steady state working point. The MPC chosen operates over infinite horizon in order to guarantee stability and it is implemented with a terminal penalty. The ID methodology considers norm based indexes for controllability, as well as robust performance conditions by using a multi-model approach. Mathematically, the ID is stated as a multiobjective nonlinear constrained optimization problem, tackled in different ways. Particularly, objective functions include investment, operating costs, and dynamical indexes based on the weighted sum of some norms of different closed loop transfer functions of the system. The paper illustrates the application of the proposed methodology with the ID of the activated sludge process of a wastewater treatment plant (WWTP).
\end{abstract}

Keywords: Integrated Design, Model Predictive Control, Terminal Penalty, Robustness, Activated Sludge Process

\section{Introduction}

During the past decades there has been an increasing awareness both in academia and industry that controllability issues need to be considered during the process design stage. Processes are more complex every time, and the advantage of considering controllability issues at the early stage of process design has been broadly recognized in the literature [1-3]. The controllability is associated with the ability of the process to achieve acceptable control performance. This measure is independent of the controller implemented, but sometimes the controllability is indicated by means of closed loop performance measurements, being dependent of the particular controller.

The classical design approach performs sequential process and control system design, with the subsequent problems in costs, performance, product quality, safety and economic benefits in general. The simultaneous process and control design, also called Integrated Design (ID), leads to a nonlinear multiobjective optimization problem where economic objectives, operability specifications and control performance are considered at design stage, giving an optimum plant more controllable.

Based on that, many authors have proposed different ID methodologies, particularly of chemical processes [4-8], because in those processes there are usually many conflicts between steady state economics and dynamic performance causing serious process synthesis difficulties. Although it is not the aim of this work (plant units and their interconnection are already fixed, i.e. process synthesis is finished), the most complete applications of simultaneous process and control design, also include the process synthesis or the control structure selection, as in the mentioned works. They involve binary decisions resulting into mixed-integer nonlinear optimization problems (MINLP), and when dynamical simulations are included, mixed-integer dynamical optimization problems (MIDO).

Most of the ID approaches use conventional PID controllers, and only few works [5], [9-11] have addressed the inclusion of advanced control techniques such as Model Based Predictive Controllers (MPC). The reason is that this control scheme implies the on-line solution of an optimization problem, leading to a drastic increase of complexity. However, the recent advances in MPC parametric controllers are spreading the use of this technique, because the explicit control law simplifies the optimization problems [12]. Recently, optimal control has also been considered in the ID procedure [13]. 
In this work, a new methodology for ID including linear MPC with terminal cost to guarantee stability is presented, following a multi-model and multiobjective approach using norm based controllability indexes. This methodology has been applied to the ID of the activated sludge process (ASP) considering two different models based on the Activated Sludge Model $n^{\circ} 1$ (ASM1); one only for organic matter removal and another with nitrification and denitrification processes [14]. Both are complex nonlinear processes with large load disturbances, making them suitable to test the ID methodology.

Some previous examples of ID applied to the ASP with PI control are given in [15], where Linear Matrix Inequality (LMI) constraints impose stability conditions and some desired closed loop behaviour, and also in [16]. In [17, 18] an ID problem considering plant costs and a sensitivity analysis methodology for the ASP was developed in order to select the best operational and control strategies. In order to improve control performance for the increasing environmental regulations, MPC was included in the activated sludge ID [9, 19, 20]. MPC has become very popular in the process industries as advanced controller because it exhibits good performance for multivariable systems and it handles constraints efficiently. Although those works present good results, the MPC implemented does not guarantee stability for all sets of tuning parameters.

In this work, the ID presented by authors in previous papers is extended to the case of MPC with guaranteed stability. The infinite horizon solution is considered, implemented as a finite horizon problem with terminal penalty, in order to solve finite dimensional QP problems. The terminal weight is obtained either from the solution of a Lyapunov equation or a Riccati equation, providing that the system is stable [20, 21]. In the second case, performance considerably improves because the final state evolution is commanded by a LQR optimal controller.

The solution of the ASP Integrated Design was usually obtained using dynamic models of the process and disturbances data [9, 10]. In those works, performance was measured by dynamical indexes such as the ISE (Integral Square Error), requiring dynamical simulations within the optimization algorithm and making the ID task extremely slow. A good alternative to speed up the ID is the use of performance indexes based on norms of some system transfer functions [23]. However, due to the use of single linearized models, some problems of stability and robustness of the closed loop system were detected in the presence of nonlinearities and load disturbances. For these reasons, multiple models and robust theory fundamentals have been included in this work to guarantee robust performance [24, 25, 26].

The performance indexes selected for ID are based on the $H_{\infty}$ and $l_{1}$ norms of different weighted closed loop transfer functions of the system, representing disturbance rejection and control efforts. Additionally, physical and operational constraints are also included to guarantee a proper working of the process. The optimization problem generated is also studied: a two-step iterative approach is compared to a single step one and the inclusion of integer parameters (MPC horizons) is tackled.

The paper is organized as follows. First, the ID methodology is presented together with the optimization procedure. Secondly the stable MPC formulation is explained, following with the controllability measures and robustness issues. Then, the ASP is presented in two versions, and the way to implement an MPC for them is explained. Finally, the ID for the ASP is stated, showing some results and conclusions.

\title{
2. Integrated Design methodology and optimization problem
}

The Integrated Design methodology allows for the evaluation of the plant parameters and the control system at the same time, designing simultaneously both the process and the controller. This problem can be conceptually posed as follows:

\author{
Minimize (Cost) \\ Subject to: \\ Differential-Algebraic Process Model, Physical and Operational Constraints, Control Scheme Equations, \\ Controllability Constraints.
}

The problem is stated mathematically as a nonlinear optimization problem with nonlinear constraints, including economic and control considerations [27]:

$$
\begin{aligned}
& \min _{\mathbf{p}, \mathbf{c}, \mathbf{x}, \mathbf{z}} J(\mathbf{x}(t), \mathbf{z}(t), \mathbf{u}(t), \mathbf{p}) \\
& \text { s.t. }
\end{aligned}
$$




$$
\begin{aligned}
& \mathbf{f}(\dot{\mathbf{x}}(t), \mathbf{x}(t), \mathbf{z}(t), \mathbf{u}(t), \mathbf{p})=0 \\
& \mathbf{h}(\mathbf{x}(t), \mathbf{z}(t), \mathbf{u}(t), \mathbf{p})=0 \\
& \mathbf{g}(\mathbf{x}(t), \mathbf{z}(t), \mathbf{u}(t), \mathbf{p}) \leq 0 \\
& \varphi(\dot{\chi}(t), \chi(t), \xi(t), \mathbf{y}(t), \mathbf{u}(t), \mathbf{c})=0 \\
& \boldsymbol{\eta}(\chi(t), \xi(t), \mathbf{y}(t), \mathbf{u}(t), \mathbf{c})=0
\end{aligned}
$$

where $\mathbf{x}(\mathrm{t})$ denotes the state variables, $\mathbf{z}(\mathrm{t})$ are the algebraic variables, $\mathbf{u}(\mathrm{t})$ are the control variables, $\mathbf{p}$ are the time invariant process design variables, $\mathbf{c}$ are the controller tuning parameters, $\mathbf{f}$ denotes the differential equations of the process (mathematical model), $\mathbf{h}$ are the algebraic equations, $\mathbf{g}$ are the inequality constraints (physical constraints, process constraints, controllability constraints, etc.), $\boldsymbol{\varphi}$ are the differential equations of the controller, $\boldsymbol{\eta}$ are the algebraic equations of the controller, $\chi$ are the differential variables of the controller, and $\xi$ are the algebraic variables of the controller. In this work, a steady state solution $\mathbf{x}_{0}$ is sought, so $\dot{\mathbf{x}}(t)=0$ in the first equation of (2), but with a small tolerance as in [16]. Then, that equation is in fact part of the inequality constraints $\mathbf{g}$ (constraints over the nonlinear differential equations of the model).

$J$ is the objective function, which can be expressed as a vector function when several objectives are present (multiobjective problem), or some of the constraints (2) are considered as objectives:

$$
\boldsymbol{J}=\left(f_{1}, \ldots, f_{i}\right) \quad i=1 \ldots O_{b} \quad\left(O_{b}=\text { number of objectives }\right)
$$

Some typical objectives are construction (investment) costs, operational costs, process controllability, operability, etc. Note that in general, dynamics are introduced in the design, but in our case the cost function does not depend on time because simulations are not considered to assess controllability. Then, the mathematical problem is analogous to (1) and (2) but with all variables defined at the steady state. The aim of the ID of process and control system problem is to obtain the optimal process design parameters $(\mathbf{p})$ and controller parameters $(\mathbf{c})$, together with a steady state working point $\left(\mathbf{x}_{0}\right)$ and related algebraic variables $(\mathbf{z})$.

The multiobjective optimization problem generated for the ID can be tackled in many ways, using deterministic or stochastic methods. For example in [28] a stochastic evolutionary strategy is used to solve the normal boundary intersection (NBI) problems that generate Pareto fronts with a uniform spread of points, or in [29] where Genetic Algorithms are used. For a review, see for example [30,31]. One approach to solve those multiobjective problems is the definition of a global function comprising the mentioned objectives:

$$
F_{t}=\sum_{i} w_{i} f_{i} \quad i=1 \ldots O_{b}
$$

where the weights $w_{i}$ give the relative importance of each objective. If all variables are normalized, all the weights are in the same scale and can be compared. Usually, there is a tradeoff between the objectives representing costs and controllability, so the weights selection must be done carefully and taking into account both aspects. Once $F_{t}$ is defined, the problem is a NLP/DAE and it is solved with an SQP (Sequential Quadratic Programming) method including the corresponding constraints.

The goal-attainment method is another way to solve those problems. This method combines all objectives including the needed constraints for that by minimizing a slack variable $\gamma$. Mathematically, if $f_{1}{ }^{*}, f_{2}{ }^{*}, f_{3}{ }^{*}$ are the goals for each objective, it is stated as follows:

$$
\min _{\mathbf{x}, \mathbf{z}, \mathbf{p}, \mathbf{c}, \gamma} \gamma
$$

s.t. $f_{i}(x)-w_{i} \gamma \leq f_{i}^{*} \quad i=1 \ldots O_{b}$

Another possibility, though not equivalent to the previous ones, consists on considering some of the objectives as constraints (typically the controllability objectives). In this work, this last approach has been considered to solve the ID, together with goal-attainment and SQP deterministic methods implemented in one or two-step iterative, as will be explained later.

\section{Model Predictive Control formulation}




\subsection{MPC with guaranteed stability}

In this paper, a linear MPC with terminal penalty has been considered to apply the ID methodology proposed. The MPC formulation consists of the on line calculation of the future control moves by solving the following constrained optimization problem subject to constraints on inputs, predicted outputs and inputs increments. The terminal penalty arises from an infinite horizon formulation, guaranteeing closed loop stability. The objective function is the following:

$$
\min _{\Delta \mathbf{u}} V(k)=\min _{\Delta \mathbf{u}}\left(\left\|\mathbf{x}\left(k+H_{c}\right)\right\|_{P}^{2}+\sum_{i=0}^{H_{c}-1}\left(\|\mathbf{x}(k+i)\|_{Q}^{2}+\|\mathbf{\Delta} \mathbf{u}(k+i)\|_{R}^{2}\right)\right)
$$

where $k$ denotes the current sampling point, $\mathbf{x}(k+i)$ is the predicted state vector at time $k+i$, depending of measurements up to time $k, \Delta \mathbf{u}$ are the changes in the manipulated variables, $H_{c}$ is the control horizon, $R$ and $Q$ are positive definite matrices representing the weights of the change of control variables and the weights of the set-point tracking errors respectively, and $P$ is a terminal penalty matrix. In this work the matrices $R$ and $Q$ are diagonal but not time dependent and the reference is fixed to zero.

The MPC prediction model is a linear discrete state space model of the plant obtained by linearizing the first-principles nonlinear model of the process [32]:

$$
\left\{\begin{array}{l}
\mathbf{x}(k+1)=A \mathbf{x}(k)+B \mathbf{u}(k)+B_{d} \mathbf{d}(k) \\
\mathbf{y}(k)=\mathbf{x}(k)
\end{array}\right.
$$

where $\mathbf{x}(\mathrm{k})$ is the state vector, $\mathbf{u}(\mathrm{k})$ is the input vector, $\mathbf{y}(\mathrm{k})$ is the output vector and $\mathbf{d}(\mathrm{k})$ the measured disturbances vector, all of them representing deviations from the steady state working point. Matrices $A, B$ and $B_{d}$ are of adequate dimensions. As usual in practice, the future measurable disturbances are kept constant in the last measured value. In this article, although full state measurement is assumed in order to test the methodology, observers based on Kalman filtering techniques could be straightforward included.

The formulation (7) is based on [21], where an infinite horizon MPC is developed with constraints on states and outputs. The feasibility of the constraints guarantees nominal stability of the closed loop system for any choice of the tuning parameters, because the objective function is a Lyapunov function. The original infinite horizon MPC objective function is the following:

$V(k)=\sum_{j=0}^{\infty}\|\mathbf{x}(k+j)\|_{Q}^{2}+\sum_{j=0}^{H_{c}-1}\|\Delta \mathbf{u}(k+j)\|_{R}^{2}=\sum_{j=H_{c}}^{\infty}\|\mathbf{x}(k+j)\|_{Q}^{2}+\sum_{j=0}^{H_{c}-1}\|\mathbf{x}(k+j)\|_{Q}^{2}+\sum_{j=0}^{H_{c}-1}\|\Delta \mathbf{u}(k+j)\|_{R}^{2}$

Considering that for sampling times $H_{c} \leq k<\infty$ the control action is constant and matrix $A$ is stable, the infinite summation can be expressed as:

$$
\sum_{j=H_{c}}^{\infty}\|\mathbf{x}(k+j)\|_{Q}^{2}=\sum_{j=0}^{\infty}\left[\left(A^{i} \mathbf{x}\left(k+H_{c}\right)\right)^{\prime} Q\left(A^{i} \mathbf{x}\left(k+H_{c}\right)\right)\right]=\mathbf{x}\left(k+H_{c}\right)^{\prime}\left[\sum_{j=0}^{\infty}\left(A^{i}\right)^{\prime} Q A^{i}\right] \mathbf{x}\left(k+H_{c}\right)=\left\|\mathbf{x}\left(k+H_{c}\right)\right\|_{P}^{2}
$$

where matrix $P$ is defined as follows:

$$
P=\sum_{j=0}^{\infty}\left(A^{j}\right)^{\prime} Q A^{j}
$$

By simple algebraic manipulation in (11), matrix $P$ can be obtained as the solution of a Lyapunov equation:

$$
P-A^{\prime} P A=Q
$$


In this way, the infinite horizon MPC objective function (9) can be expressed as (7) and the optimization can be solved as a QP problem. A sequence of control increments $\Delta \mathbf{u}(k+i)$ is obtained, but only the first element of the sequence is applied to the system at time $k+1$. The optimization problem (7) is repeated at the next sampling time in a receding horizon strategy.

The use of this terminal penalty guarantees MPC stability, but for slow response processes such as the activated sludge process, general performance deteriorates because of the free response from sampling time $H_{c}$. For that reason, in this article an unconstrained LQR (Linear Quadratic Regulator) controller has been considered from sampling time $H_{c}$ to infinity [22], improving performance significantly.

$$
\begin{aligned}
& \mathbf{u}(k+j)=-K \mathbf{x}(k+j), \quad H_{c} \leq k<\infty \\
& K=\left(B^{\prime} P B+R\right)^{-1} B^{\prime} P A \\
& P=A^{\prime} P A-A^{\prime} P B\left(B^{\prime} P B+R\right)^{-1} B^{\prime} P A+Q
\end{aligned}
$$

where $K$ and $P$ are the solution of the following unconstrained infinite horizon LQR problem with weights $Q$ and $R$ :

$$
\min _{K} V_{L Q}=\sum_{j=H_{c}}^{\infty}\left(\|\mathbf{x}(k+j)\|_{Q}^{2}+\|\mathbf{u}(k+j)\|_{R}^{2}\right)
$$

Considering the matrix $P$ obtained from Riccati equation (13), the term $\left\|\mathbf{x}\left(k+H_{c}\right)\right\|_{P}^{2}$ in (7) represents the settling cost of the system from $H_{c}$ to infinity under this type of control. Therefore, this term is added to $V(k)$ in order to compute the total cost.

\subsection{MPC basic relationships}

The MPC control law can be expressed in the Laplace domain for the SISO case as: (Fig. 1)

$$
u(s)=K_{1}(r(s)-y(s))+K_{2} d(s)
$$

where $K_{1}$ is the transfer function between the control signal and the error signal $(e(s)=r(s)-y(s))$ and $K_{2}$ is the transfer function between the control signal and $d(s)$. Both transfer functions depend on the control system tuning parameters $\left(Q, R, H_{c}\right)$ and on plant parameters. This linear control law is obtained by solving the unconstrained optimization problem (7) [32]. For simplicity, only the SISO case is presented, but when several inputs, disturbances or outputs are present, the closed loop sensitivity functions can be calculated analogously. In this work, only $R$ and $H_{c}$ are tuned, because variations in $Q$ produce similar effects on performance. Note that the proposed MPC structure is a combined feedforward-feedback system.

Taking into account control law (15) and the transfer function of the open loop system, the closed loop response can be obtained from:

$$
y(s)=\frac{G_{0} K_{1}}{1+G_{0} K_{1}} r(s)+\frac{1}{1+G_{0} K_{1}} \tilde{d}(s)
$$

where $\tilde{d}(s)$ are the filtered disturbances $\tilde{d}(s)=\left(G_{0} K_{2}+G d_{0}\right) d(s)$, and the nominal transfer functions are denoted by $G_{0}$ and $G d_{0}$.

Equation (16) can be expressed substituting the sensitivity function and complementary sensitivity functions:

$$
y(s)=T_{0}(s) r(s)+S_{0}(s) R_{d 0}(s) d(s)
$$

where 


$$
\begin{gathered}
S_{0}=\frac{1}{1+G_{0} K_{1}} ; T_{0}=\frac{G_{0} K_{1}}{1+G_{0} K_{1}} \\
R_{d 0}(s)=G_{d 0}(s)-K_{2} G_{0}(s)
\end{gathered}
$$

In order to state the ID problem for the nominal case, the sensitivity function $S_{0}(s) R_{d 0}(s)$ between the load disturbances and the outputs will be considered, as well as the control sensitivity transfer function $M_{0}(s)$ between the load disturbances and the control signals when the reference is set to zero. Its calculation is straightforward applying block algebra to diagram of Fig. 1:

$$
M_{0}(s)=\frac{u(s)}{d(s)}=\frac{K_{2}-K_{1} G_{d 0}}{1+G_{0} K_{1}}
$$

The ID methodology is stated in this work for an infinite horizon MPC (with penalty term), although it is fully applicable to the case of a simple finite horizon MPC (without penalty term). The procedure for calculating the sensitivity transfer functions is the same, changing only the objective function (7) [23].

\section{Controllability indexes}

The selection of appropriate controllability indexes is very important in order to state the ID problem. Dynamic indexes such as the ISE (Integral Square Error) are interesting because they consider all nonlinearities of the process, but their calculation is rather slow (they need simulations), increasing considerably the computational time, and ignoring the magnitude of deviations from the reference. For that reasons, indexes based on the $\mathrm{H}_{\infty}$ and $l_{1}$ norms of the closed loop sensitivity functions of the system are considered here [23]. Although those functions are only defined for linear control systems, it can be shown that they are also valid when the set of MPC active constraints is fixed [32, 33]. This assumption may be too strict, and for that reason the constraints (26) are used to keep the variables within the feasibility region.

\subsection{Mixed sensitivity index}

The first index proposed here is an $\mathrm{H}_{\infty}$ mixed sensitivity function that takes into account both disturbance rejection and control effort objectives (transfer functions dependence on Laplace variable $s$ has been omitted for brevity):

$$
\left\|N_{0}\right\|_{\infty}=\max _{\omega}\left|N_{0}(j \omega)\right|
$$

where $\quad N_{0}=\left(\begin{array}{l}W_{p} \cdot S_{0} \cdot R_{d 0} \\ W_{\text {esf }} \cdot s \cdot M_{0}\end{array}\right)$

This index is extensively used in robust control design [24, 32] and the objective is to minimize its value. $W_{p}$ and $W_{\text {esf }}$ are suitable weights to achieve closed loop performance specifications and to reduce the control efforts respectively. Note that control efforts rather than magnitudes of control are included in the objective function by considering the derivative of the transfer function $M_{0}$.

\subsection{Performance constraints}

In order to ensure disturbance rejection (considering normalized disturbances), the following equation must be satisfied in the disturbances frequency range:

$\left|S_{0}(j \omega) \cdot R_{d 0}(j w)\right| \cdot|d(\omega)|<1 \quad \omega \in\left(\omega_{1}, \omega_{2}\right)$

where $S_{0}(j \omega)$ is the frequency response of the sensitivity function, $d(\omega)$ is the disturbance spectra, and $\left(\omega_{1}, \omega_{2}\right)$ is the disturbances frequency range. By choosing a weight $W_{p}(s)$ satisfying

$20 \cdot \log \left|W_{p}(j \omega)\right|^{-1}<20 \cdot \log |d(\omega)|^{-1}$ 
disturbance rejection can be ensured, imposing the following constraint in the optimization ID procedure:

$\left\|W_{p} \cdot S_{0} \cdot R_{d 0}\right\|_{\infty}<1$

A typical choice for the weight $W_{p}(s)$ is a rational function with one zero and one pole.

\subsection{Control and output bounds}

The maximum deviation value of the control $\left(u_{\max }\right)$ and output variables $\left(y_{\max }\right)$, for the worst case of disturbances, is constrained to be less than certain limits by means of the $l_{1}$ norm and the following conditions for normalized disturbances, in order to avoid saturations and to keep the control system in the linear region:

$\left\|M_{0}\right\|_{1}<u_{\max } ;\left\|S_{0} \cdot R_{d 0}\right\|_{1}<y_{\max }$

This worst case index seems to be suitable for this process because disturbances are not fully predictable and a slackening of that condition could saturate the actuator. The $l_{1}$ norm of a stable transfer function such as $M_{0}$ is defined as follows:

$$
\left\|M_{0}\right\|_{1}=\max _{d(t)} \frac{\|u(t)\|_{\infty}}{\|d(t)\|_{\infty}}
$$

\subsection{Other indexes for controllability}

Finally, other quantities based on norms of the system sensitivity functions have been applied. Particularly interesting to quantify the disturbance rejection is the $l_{1}$ norm of the transfer function that filters the measurable disturbances (see equation (16) and recall that $\left.\tilde{d}(s)=\left(G_{0} K_{2}+G_{d 0}\right) d(s)\right)$ :

$$
\left\|G_{0} K_{2}+G_{d 0}\right\|_{1}=\max _{d(t)} \frac{\|\tilde{d}(t)\|_{\infty}}{\|d(t)\|_{\infty}}
$$

If this index is minimized, the effect of disturbances on the process output is also minimized, for the worst combination of disturbances.

\section{Robust Integrated Design}

Robustness issues have been considered in the ID, assuming that there is a nominal model of the process and that the corresponding uncertainty limits take into account nonlinearities in the real plant. The first step is the selection of the region around the nominal point in which performance specifications are going to be satisfied. In this work, polytope uncertainty is considered, so two families of models $\Pi_{u}$ and $\Pi_{d}$ are determined by means of multiple linear local models (vertices of the polytope).

In the presence of uncertainty, the robust performance is attained if for each $d(t) \in \mathcal{L}_{2}[0, \infty)$, the desired performance condition is satisfied for every perturbed model $G \in \Pi_{u}$ and $G_{d} \in \Pi_{d}$. This sufficient condition for robust disturbance rejection can be stated analogously to the nominal case (25):

$$
\left\|W_{p} \cdot S \cdot R_{d}\right\|_{\infty}<1
$$

where $R_{d}$ represents the open loop residual disturbance remaining after the feedforward compensation and due to the uncertainty.

$$
R_{d}(s)=G_{d}(s)-K_{2} G(s)
$$


In this work, multiple linear models belonging to the families $\Pi_{u}$ and $\Pi_{d}$ have been considered for the robust ID, and the plant and MPC obtained are optimal in the region they define. At each iteration of the ID optimization, open loop transfer functions $G(s)$ and $G_{d}(s)$ for each local model (including the nominal) are calculated by linearization, and the sensitivity functions $S(s) \cdot R_{d}(s)$ are also calculated with the current controller values (c). Then, for every local model, constraint (29) must be imposed to guarantee robust performance.

\section{Description of the activated sludge process and control system}

\subsection{Wastewater treatment plant description}

The purpose of the wastewater treatment plants is to process sewage and return clean water to the river, and the ASP is a very important part of the cleaning procedure. The water treatment comprises the following basic steps, though in this work only b) and c) are considered:

a) The primary treatment is dedicated to the removal of gross solids, sand, oil and grease. A primary sedimentation is the last step of this stage. This process removes up to $50 \%$ of the total polluting sewage load.

b) The secondary treatment is the ASP. The mixed outlet stream from the primary sedimentation tanks is passed to the reactor. There, the aerobic action of a mixture of microorganisms is used to reduce the substrate concentration in the water. A bacterial culture degrades the organic substrate converting it into inorganic products, more biomass and water. The dissolved oxygen required is provided by a set of aeration turbines.

c) Clarification. The effluent is feed into clarification tanks, where the activated sludge and clean water are separated. After this, the water contains approximately $10 \%$ of the waste material and the water is discharged to the river. Between $25 \%$ and $100 \%$ of the settled activated sludge is recycled to re-inoculate the reactor.

\subsection{Mathematical model of the ASP for substrate removal and control problem (case study I)}

A simple model of ASP only for organic matter (substrate) elimination is described first. The plant and controller layout can be seen in Fig. 2, comprising a bioreactor and a settler for clarification. The mathematical model based on mass balances is [35]: (assuming perfectly mixed tanks)

\section{- Aeration tanks}

The rate of change of the biomass, organic substrate and dissolved oxygen concentrations are given by:

$$
\begin{aligned}
\frac{d x_{1}}{d t} & =\mu_{\max } y_{c} \frac{s_{1} x_{1}}{\left(K_{s}+s_{1}\right)}-K_{d} \frac{x_{1}^{2}}{s_{1}}-K_{c} x_{1}+\frac{q}{V_{1}}\left(x_{i r}-x_{1}\right) \\
\frac{d s_{1}}{d t} & =-\mu_{\max } \frac{s_{1} x_{1}}{\left(K_{s}+s_{1}\right)}+f k_{d} K_{d} \frac{x_{1}^{2}}{s_{1}}+f k_{d} K_{c} x_{1}+\frac{q}{V_{1}}\left(s_{i r}-s_{1}\right) \\
\frac{d c_{1}}{d t} & =K_{l a} f k_{1}\left(c_{s}-c_{1}\right)-O U R-\frac{q}{V_{1}} c_{1}
\end{aligned}
$$

where $x_{1}, s_{1}$ and $c_{1}$ are the biomass, substrate (Chemical Oxygen Demand (COD)) and dissolved oxygen (DO) concentration at the output of the aeration tanks (mg/l); $x_{i r}$ and $s_{i r}$ are respectively the inlet biomass and substrate (mg/l). $\mu_{\max }$ is the maximum growth rate of the microorganisms, $q$ is the inlet flow $\left(\mathrm{m}^{3} / \mathrm{h}\right), K_{d}$ is the kinetic coefficient of biomass decay by endogenous metabolism $(1 / \mathrm{h}), K_{c}$ is the kinetic coefficient of biomass decay by biological waste, $V_{1}$ is the total useful volume for the six aeration tanks $\left(\mathrm{m}^{3}\right), y_{c}$ is the yield coefficient between cellular growth and substrate elimination, $f k_{d}$ is the yield coefficient between biomass endogenous and substrate contribution to the medium, $c_{s}$ is the DO concentration at saturation, $K_{l a}$ is the mass transfer coefficient, $f k_{1}$ is the aeration factor which depends on the number and speed of working turbines, OUR is the oxygen uptake rate and $K_{s}$ is the saturation constant.

For the rate of change of the biomass, the first term describes the biomass growth according to the Monod model, the second describes cell death, the third describes the biological waste, and the final term quantifies the dilution effects. For the rate of consumption of organic substrate, the first term expresses the decrease of the substrate through the activity of the biomass 
(Monod model), the second and third ones describe the transformation part of the dead biomass and biological waste into organic substrate, and the last term is the difference between the input and output substrate mass flows. Finally, for the dissolved oxygen concentration, the equation follows the classic literature: the first term is the rate of oxygen transferred to the water, the second describes the rate of oxygen used by the microorganisms (uptake rate), and the final term quantifies the dilution effects.

Algebraic equations for $x_{i r}$ and $s_{i r}$ are expressed as mass balances:

$x_{i r}=\frac{x_{i} q_{i}+x_{r} q_{r}}{q} \quad s_{i r}=\frac{s_{i} q_{i}+s_{1} q_{r}}{q}$

where $x_{i}, s_{i}$ are the biomass and substrate at the influent, $q_{i}$ is the input flow to the process. $s_{r} x_{r}$ and $q_{r}$ are the recycled concentrations and flow.

Equation for oxygen uptake rate is:

OUR $=-K_{01} \mu_{\max } \frac{x_{1} \cdot s_{1}}{\left(K_{s}+s_{1}\right)}$

where $K_{01}$ is the yield coefficient between the cellular growth and the oxygen consumption rate.

\section{- Secondary clarifiers (settlers)}

The operation of these elements is described by mass balance equations and one expression for the settling of activated sludge. The model takes into account the difference in settling rates between layers of increasing biomass concentration. This model attempts to capture the dynamic behavior of the clarifiers:

$$
\begin{aligned}
& A \cdot l_{d} \frac{d x_{d}}{d t}=q_{s a l} x_{b}-q_{s a l} x_{d}-A \cdot v_{s}\left(x_{d}\right) \\
& A \cdot l_{b} \frac{d x_{b}}{d t}=q x_{1}-q_{s a l} x_{b}-q_{2} x_{b}+A \cdot v_{s}\left(x_{d}\right)-A \cdot v_{s}\left(x_{b}\right) \\
& A \cdot l_{r} \frac{d x_{r}}{d t}=q_{2} x_{b}-q_{2} x_{r}+A \cdot v_{s}\left(x_{b}\right)
\end{aligned}
$$

where $x_{d}$ is the biomass concentration at the surface of the settler leaving the plant, $q_{\text {sal }}$ is the flow of clean water at the output of the settler, $x_{b}$ is the biomass concentration in the second layer, $q_{2}$ is the activated sludge total recycling flow, $x_{r}$ is the biomass concentration at the bottom of the settler, $v_{s}$ is the settling rate of the activated sludge, $A$ is the area of the settler, and $l_{d}, l_{b}, l_{r}$ are the height of the first, second and third layer, respectively (Fig. 2). Note that the settler input flow $q$ enters to the unit at the second layer level.

The settling rate is calculated experimentally, the parameters are evaluated to fit a curve defined by experimental points:

$v_{s}\left(x_{b}\right)=n r \cdot x_{b} \cdot \mathrm{e}^{\left(-a r \cdot x_{b}\right)} ; v_{s}\left(x_{d}\right)=n r \cdot x_{d} \cdot \mathrm{e}^{\left(-a r \cdot x_{d}\right)}$

The relations between the different flows are:

$$
q=q_{i}+q_{r} ; \quad q_{s a l}=q_{i}-q_{p} ; \quad q_{2}=q_{r}+q_{p}
$$

where $q_{p}$ is the purge flow.

Unknown parameters in the differential equations are calculated to minimize a function expressed as the difference between the model output and real data from a plant (located in Manresa, Spain) when the same inputs are applied to both systems. A whole description of parameters is given in [35]. 
The control of this process aims to keep the substrate at the output $\left(s_{1}\right)$ below a legal value despite the large variations of the flow rate $\left(q_{i}\right)$ and the substrate concentration of the incoming water $\left(s_{i}\right)$. The disturbances vector is: $\mathbf{d}=\left(s_{i}, q_{i}\right)$. The recycling flow $\left(q_{r}\right)$ is the manipulated variable and the controlled output is the substrate $\left(s_{1}\right)$ in the reactor: $u(k)=q_{r} ; y(k)=s_{1}($ Fig. 2). Biomass $\left(x_{1}\right)$ in the reactor is a bounded variable. Different sets of disturbances have been considered from COST 682 program and its benchmark [36], and particularly the set for storm weather (Fig. 3). In the proposed ID methodology, dynamic simulations are not performed, so disturbance sets are used only for normalizing the $l_{1}$ norms and for simulations after the plant has been designed.

In the MPC the following dynamic constraints are imposed:

$$
\begin{aligned}
& S_{1 d}<s_{1}<s_{1 u} \\
& x_{1 d}<x_{1}<x_{1 u} \\
& q_{r d}<q_{r}<q_{r u} \\
& \Delta q_{r d}<\Delta q_{r}<\Delta q_{r u}
\end{aligned}
$$

where $s_{1 d}, s_{1 u} ; x_{1 d}, x_{1 u} ; q_{r d}, q_{r u} ; \Delta q_{r d}, \Delta q_{r u}$ are the bounds for substrate, biomass, recycling flow and the increments of recycling flow respectively.

\subsection{Mathematical model of the ASP for nitrogen removal and control problem (case study II)}

The full ASP is based on the ASM1 model [36], which includes organic matter and nitrogen removal. However, the ASM1 is a very complex model, strongly nonlinear, making it difficult to use in the ID methodology. For that reason, a simplified model is considered, where the variables with slow variation are considered constant and the ones that change fast are neglected [14]. After the simplification, from the eight processes modeled by the ASM1, only three are considered.

The plant layout consists of two reactors, an anoxic tank followed by an aerated one, and a secondary settler (Fig. 4). The first step in the nitrogen removal process is the aerobic nitrification where the microorganisms nitrificators convert the ammonium into nitrate, and then this step is followed by an anoxic one (denitrification), where the nitrate is transformed in gaseous ammonium which is eliminated from the water in the atmosphere. For this process the microorganisms use the readily biodegradable matter. For satisfying the need of readily degradable organic matter form the denitrification process, the anoxic reactor is located in the first place to use the organic matter from the influent. Two recycling flows are added, one internal to recirculate nitrate to the first reactor, and one external to recirculate sludge from the setter (Fig. 4).

In this model six basic components in the water are considered for each reactor (aerated and anoxic), respectively: autotrophic biomass $X_{A, \text { nit }}, X_{A, \text { dnit }}$; heterotrophic biomass $X_{H, n i t}, X_{H, d n i t}$; readily biodegradable substrate $S_{S, n i t}$, $S_{S, \text { dnit }}$; soluble nitrate nitrogen $S_{N O, \text { nit }}, S_{N O \text {,dnit }}$; soluble ammonium nitrogen $S_{N H, \text { nit }}, S_{N H, d n i t}$; and dissolved oxygen $S_{O, \text { nit }}$, $S_{O, \text { dnit }}$. For the settler $X_{\text {rec }}$ is the concentration of recycled biomass. The non linear differential equations (mass balances) for the two reactors and settler are the following, where the whole set of numerical values for the coefficients are defined in [14].

$$
\begin{aligned}
& \dot{X}_{A, \text { nit }}=\left(1+r_{1}+r_{2}\right) D_{\text {nit }}\left(X_{A, \text { dnit }}-X_{A, \text { nit }}\right)+\left(\mu_{A, \text { nit }}-b_{A}\right) X_{A, \text { nit }} \\
& \dot{X}_{H, \text { nit }}=\left(1+r_{1}+r_{2}\right) D_{n i t}\left(X_{H, \text { dnit }}-X_{H, n i t}\right)+\left(\mu_{H, n i t}-b_{H}\right) X_{H, \text { nit }} \\
& \dot{S}_{S, \text { nit }}=\left(1+r_{1}+r_{2}\right) D_{n i t}\left(S_{S, \text { nnit }}-S_{S, n i t}\right)-\left(\mu_{H, n i t}-\mu_{H a, n i t}\right) \frac{X_{H, n i t}}{Y_{H}} \\
& \dot{S}_{N H, \text { nit }}=\left(1+r_{1}+r_{2}\right) D_{n i t}\left(S_{N H, \text { dnit }}-S_{N H, \text { nit }}\right)-\left(i_{X B}+\frac{1}{Y_{A}}\right) \mu_{A, \text { nit }} X_{A, \text { nit }}-\left(\mu_{H, n i t}+\mu_{H a, n i t}\right) i_{X B} X_{H, n i t} \\
& \dot{S}_{N O, \text { nit }}=\left(1+r_{1}+r_{2}\right) D_{n i t}\left(S_{N O, \text { dnit }}-S_{N O, \text { nit }}\right)+\mu_{A, \text { nit }} \frac{X_{A, n i t}}{Y_{A}}-\frac{1-Y_{H}}{2.86 Y_{H}} \mu_{H a, n i t} X_{H, n i t} \\
& \dot{S}_{O, \text { nit }}=\left(1+r_{1}+r_{2}\right) D_{\text {nit }}\left(S_{O, \text { dnit }}-S_{O, \text { nit }}\right)+a_{0} Q_{a i r}\left(C_{S}-S_{O, n i t}\right)-\frac{4.57-Y_{A}}{Y_{A}} \mu_{A, \text { nit }} X_{A, \text { nit }}-\frac{1-Y_{H}}{Y_{H}} \mu_{H a, n i t} X_{H, \text { nit }} \\
& \dot{X}_{A, \text { dnit }}=D_{\text {dnit }}\left(X_{A, \text { in }}+r_{1} X_{A, \text { nit }}\right)+\alpha r_{2} D_{\text {dnit }} X_{\text {rec }}-\left(1+r_{1}+r_{2}\right) D_{d n i t} X_{A, \text { dnit }}+\left(\mu_{A, \text { dnit }}-b_{A}\right) X_{A, \text { dnit }} \\
& \dot{X}_{H, \text { dnit }}=D_{\text {dnit }}\left(X_{H, \text { in }}+r_{1} X_{H, \text { nit }}\right)+(1-\alpha) r_{2} D_{\text {dnit }} X_{r e c}-\left(1+r_{1}+r_{2}\right) D_{\text {dnit }} X_{H, \text { dnit }}+\left(\mu_{H, \text { dnit }}-b_{H}\right) X_{H, \text { dnit }}
\end{aligned}
$$




$$
\begin{aligned}
& \dot{S}_{S, \text { dnit }}=-\left(\mu_{H, \text { dnit }}-\mu_{H a, d n i t}\right) \frac{X_{H, d n i t}}{Y_{H}}+D_{d n i t}\left(S_{S, \text { in }}+r_{1} S_{S, \text { nit }}\right)-\left(1+r_{1}+r_{2}\right) D_{d n i t} S_{S, \text { dnit }} \\
& \dot{S}_{N H, \text { dnit }}=D_{\text {dnit }}\left(S_{N H, \text { in }}+r_{1} S_{N H, \text { nit }}\right)-\left(1+r_{1}+r_{2}\right) D_{\text {dnit }} S_{N H, \text { dnit }}-\left(\mu_{H, d n i t}+\mu_{H a, d n i t}\right) i_{X B} X_{H, \text { dnit }}-\left(i_{X B}+\frac{1}{Y_{A}}\right) \mu_{A, \text { dnit }} X_{A, \text { dnit }} \\
& \dot{S}_{N O, \text { dnit }}=D_{\text {dnit }}\left(S_{N O, \text { in }}+r_{1} S_{N O, \text { nit }}\right)-\left(1+r_{1}+r_{2}\right) D_{d n i t} S_{N O, d n i t}+\mu_{A, \text { dnit }} \frac{X_{A, \text { dnit }}}{Y_{A}}-\frac{1-Y_{H}}{2.86 Y_{H}} \mu_{H a, d n i t} X_{H, \text { dnit }} \\
& \dot{S}_{O, \text { dnit }}=0 \\
& \dot{X}_{r e c}=\left(1+r_{2}\right) D_{d e c}\left(X_{A, n i t}+X_{H, n i t}\right)-\left(r_{2}+w\right) D_{d e c} X_{r e c}
\end{aligned}
$$

The growth rates of autotrophs and heterotrophs in aerobic conditions are $\mu_{A, n i t}, \mu_{H, \text { nit }}$; the growth rate of heterotrophs in anoxic conditions is $\mu_{\mathrm{Ha}, \text {,nit }}$, and the analogous growth rates for the anoxic reactor are $\mu_{A, d n i t}, \mu_{H, d n i t}$ and $\mu_{H a, d n i t}$ :

$$
\begin{aligned}
& \mu_{A, \text { nit }}=\mu_{\text {max }, A} \frac{S_{N H, \text { nit }}}{\left(K_{N H, A}+S_{N H, \text { nit }}\right)} \frac{S_{O, \text { nit }}}{\left(K_{O, A}+S_{O, \text { nit }}\right)} ; \mu_{H, \text { nit }}=\mu_{\max , H} \frac{S_{S, \text { nit }}}{\left(K_{S}+S_{S, n i t}\right)} \frac{S_{N H, \text { nit }}}{\left(K_{N H, H}+S_{N H, n i t}\right)} \frac{S_{O, \text { nit }}}{\left(K_{O, H}+S_{O, n i t}\right)} \\
& \mu_{\mathrm{Ha}, \text { nit }}=\mu_{\max , H} \frac{S_{S, \text { nit }}}{\left(K_{S}+S_{S, \text { nit }}\right)} \frac{S_{N H, \text { nit }}}{\left(K_{N H, H}+S_{N H, \text { nit }}\right)} \frac{S_{O, \text { nit }}}{\left(K_{O, H}+S_{O, \text { nit }}\right)} \frac{S_{N O, \text { nit }}}{\left(K_{N O}+S_{N O, \text { nit }}\right)} \eta_{N O} \\
& \mu_{A, \text { dnit }}=\mu_{\max , A} \frac{S_{N H, \text { dnit }}}{\left(K_{N H, A}+S_{N H, d n i t}\right)} ; \quad \mu_{H, \text { dnit }}=\mu_{\max , H} \frac{S_{S, \text { dnit }}}{\left(K_{S}+S_{S, \text { dnit }}\right)} \frac{S_{N H, \text { dnit }}}{\left(K_{N H, H}+S_{N H, \text { dnit }}\right)} \\
& \mu_{\mathrm{Ha}, \text { dnit }}=\mu_{\mathrm{max}, \mathrm{H}} \frac{S_{S, \text { dnit }}}{\left(K_{S}+S_{S, \text { dnit }}\right)} \frac{S_{N H, \text { dnit }}}{\left(K_{N H, H}+S_{N H, \text { dnit }}\right)} \frac{S_{N O, \text { dnit }}}{\left(K_{N O}+S_{N O, \text { dnit }}\right)} \eta_{N O}
\end{aligned}
$$

Flow ratios between external recycling flow $\left(Q_{r 2}\right)$, internal recycling flow $\left(Q_{r 1}\right)$, purge flow $\left(Q_{w}\right)$ and the influent flow $\left(Q_{i n}\right)$ are the following, together with the dilution rates $D_{\text {nit }}, D_{\text {dnit }}, D_{\text {dec }}$ :

$$
r_{1}=\frac{Q_{r 1}}{Q_{i n}} ; r_{2}=\frac{Q_{r 2}}{Q_{i n}} ; w=\frac{Q_{w}}{Q_{i n}} ; D_{\text {nit }}=\frac{Q_{i n}}{V_{n i t}} ; D_{d n i t}=\frac{Q_{i n}}{V_{d n i t}} ; D_{d e c}=\frac{Q_{i n}}{V_{d e c}}
$$

For this process, the control objective considered is to keep the nitrate in the anoxic tank $\left(S_{N O \text {,dnit }}\right)$ below legal limits, manipulating $Q_{r 1}$. The set of load disturbances $\left(S_{s, i n}, Q_{i n}\right)$ are identical to those in Fig. 3, only changing the stationary level. The $Q_{r 2}$ is kept constant, because it has no relevant influence in the $S_{N O \text {,dnit }}$. The MPC dynamic constraints imposed in this case are:

$$
\begin{aligned}
& S_{N O, d n i t, d}<S_{N O, d n i t}<S_{N O, d n i t, u} \\
& Q_{r 1 d}<Q_{r 1}<Q_{r 1 u} \\
& \Delta Q_{r 1 d}<\Delta Q_{r 1}<\Delta Q_{r 1 u}
\end{aligned}
$$

where $S_{N O, d n i t, d}, S_{N O, d n i t, u} ; Q_{r 1 d}, Q_{r 1 u} ; \Delta Q_{r 1 d}, \Delta Q_{r 1 u}$ are the bounds for nitrate, internal recycling flow and its increments respectively.

\section{Integrated Design (ID) of the activated sludge processes and MPC controller}

The ID problem consists of simultaneously determining the plant and MPC controller parameters together with a steady state working point, while the investment and operating costs are minimized. It is interesting to apply ID to the ASP because with only a classical steady state design, disturbance rejection is usually poor when the controller is implemented afterwards. If controllability issues are considered at the design stage, the plant performance increases considerably.

\subsection{Optimization problem for case study I}


The objective function $\boldsymbol{J}$ for the ID of the ASP can be expressed as $\boldsymbol{J}=\left(f_{1}, f_{2}\right)$, where $f_{1}$ represents the construction (plant dimensions) and operational costs (pumping energy) [9], and $f_{2}$ represents the process controllability. The mixed sensitivity index of equation (21) is chosen as main controllability index for the reasons already explained. The case study I considers ASP process of 6.2 (only substrate removal), and the objectives are:

$$
\begin{aligned}
& f_{1}(\mathbf{p}, \mathbf{c}, \mathbf{x}, \mathbf{z})=w_{1} \cdot V_{1 n}^{2}+w_{2} \cdot A_{n}^{2}+w_{3} \cdot q_{2}^{2} \\
& f_{2}(\mathbf{p}, \mathbf{c}, \mathbf{x}, \mathbf{z})=\|N\|_{\infty}
\end{aligned}
$$

where $V_{1 n}$ and $A_{n}$ are the normalized values for the reactor volume and the cross-sectional area of the settler, $q_{2}$ is the total recycling flow, $\mathbf{p}=\left(V_{1}, A\right), \mathbf{x}=\left(s_{1}, x_{1}, c_{1}, x_{d}, x_{b}, x_{r}\right), \mathbf{z}=\left(q_{r}, q_{p}\right)$ and $\mathbf{c}=\left(R, H_{c}\right)$ are the optimization variables, and $w_{i}=1(i=1,2,3)$.

For a right functioning of the ASP, the following process and controllability constraints are included, together with physical bounds for all variables:

- $\quad$ Residence time and mass load in the reactor limited between $\left[\mathrm{ml}_{d}, \mathrm{ml}_{u}\right]$ and $\left[\mathrm{ret}_{d}, \mathrm{ret}_{u}\right]$ :

$$
r e t_{d} \leq \frac{V_{1}}{q} \leq r e t_{u} ; m l_{d} \leq \frac{q_{i} \cdot s_{i}+q_{r} \cdot s_{1}}{V_{1} \cdot x_{1}} \leq m l_{u}
$$

- $\quad$ Limits in hydraulic capacity of the settler $[c h]$ and sludge age $\left[s a_{d}, s a_{u}\right]$ :

$$
\frac{q}{A} \leq c h ; s a_{d} \leq \frac{V_{1} \cdot x_{1}+A_{d} \cdot l_{r} \cdot x_{r}}{q_{p} \cdot x_{r} \cdot 24} \leq s a_{u}
$$

- $\quad$ Limits in the ratios between the input and recycled flows $\left[r e c_{d}, r e c_{u}\right]$ and between the recycled and purge flows [purg $g_{d}$ $\left.\operatorname{purg}_{u}\right]$ :

$$
\operatorname{rec}_{d} \leq \frac{q_{2}}{q_{i}} \leq \text { rec }_{u} ; \quad \operatorname{purg}_{d} \leq \frac{q_{p}}{q_{2}} \leq \text { purg }_{u}
$$

- Constraints on the nonlinear differential equations of the process to obtain a solution close to a steady state and thus to make possible the calculation of linearized models for the sensitivity transfer functions. Particularly, all normalized derivates are constrained to be less than a tolerance of $10^{-5}$.

- Controllability constraints (26) and (29), for robust performance considering a set of multiple models defined around the nominal one.

\subsection{Optimization problem for case study II}

For the process model of point 6.3 (case study II, with nitrogen removal) the function $\boldsymbol{J}=\left(f_{1}, f_{2}\right)$ is expressed as:

$$
\begin{aligned}
& f_{1}(\mathbf{p}, \mathbf{c}, \mathbf{x}, \mathbf{z})=w_{1} \cdot V_{n i t, n}^{2}+w_{1} \cdot V_{d n i t, n}^{2}+w_{3} \cdot V_{d e c, n}^{2}+w_{4} \cdot Q_{2}{ }^{2} \\
& f_{2}(\mathbf{p}, \mathbf{c}, \mathbf{x}, \mathbf{z})=\|N\|_{\infty}
\end{aligned}
$$

where $V_{n i t, n}, V_{d n i t, n}$ and $V_{d e c, n}$ are the normalized values for the volume of the reactors and settler, $Q_{2}=Q_{r 1}+Q_{r 2}+Q_{w}$ is the total recycling flow, $\mathbf{p}=\left(V_{\text {nit }}, V_{\text {dnit }}, V_{\text {dec }}\right), \mathbf{x}=\left(X_{A, n i t}, X_{H, \text { nit }}, S_{S, n i t}, S_{N H, n i t}, S_{N O, n i t}, X_{A, \text { dnit }}, X_{H, \text { dnit }}, S_{S, \text { dnit }}, S_{N H \text {,dnit }}, S_{N O \text {,dnit }}, S_{O, \text { nit }}, X_{\text {rec }}\right)$, $\mathbf{z}=\left(Q_{r 1}, Q_{r 2}, Q_{w}\right)$ and $\mathbf{c}=\left(R, H_{c}\right)$ are the optimization variables, and $w_{i}=1(i=1, \ldots, 4)$.

For the same reasons as in case study $I$, the following constraints are included:

- Process and controllability together with physical bounds for all variables:

$$
\begin{aligned}
\text { ret }_{d} & \leq \frac{V_{\text {dnit }}}{Q_{i n}+Q_{r 1}+Q_{r 2}} \leq \text { ret }_{u} ; \text { ret }_{d} \leq \frac{V_{\text {nit }}}{Q_{i n}+Q_{r 1}+Q_{r 2}} \leq \text { ret }_{u} \\
m l_{d} & \leq \frac{Q_{i n} S_{s, \text { in }}+Q_{r 1} S_{s, n i t}+Q_{r 2} S_{s, n i t}}{V_{\text {dnit }} \cdot X_{H, \text { dnit }}} \leq m l_{u} ; m l_{d} \leq \frac{\left(Q_{i n}+Q_{r 1}+Q_{r 2}\right) \cdot S_{s, \text { dnit }}}{V_{n i t} \cdot X_{H, n i t}} \leq m l_{u}
\end{aligned}
$$




$$
\begin{aligned}
& \frac{Q_{\text {in }}+Q_{r 1}+Q_{r 2}}{V_{\text {dec }}} \leq \text { ch } ; \operatorname{sa}_{d} \leq \frac{V_{\text {dnit }} \cdot\left(X_{A, \text { dnit }}+X_{H, \text { dnit }}\right)+V_{\text {nit }} \cdot\left(X_{A, \text { nit }}+X_{H, n i t}\right)+V_{\text {dec }} X_{\text {rec }}}{Q_{w} \cdot X_{\text {rec }}} \leq s a_{u} \\
& \text { rec }_{d} \leq \frac{Q_{r 2}+Q_{w}}{Q_{\text {in }}} \leq \text { rec }_{u} ; \operatorname{purg}_{d} \leq \frac{Q_{w}}{Q_{r 2}+Q_{w}} \leq \operatorname{purg}_{u}
\end{aligned}
$$

- Constraints over the nonlinear differential equations of the process, analogous to the previous case, and controllability constraints (25) for nominal performance.

\subsection{Optimization strategies}

When the ID problem includes an MPC, as it is the case with this work, the existence of real and integer parameters (control horizon) make necessary the development of particular optimization strategies. The fact that controller tuning and plant specific design are relatively independent problems together with the complexity of the problem due to plant nonlinearities both make it interesting to use a two-step iterative algorithm with some variants detailed below.

\section{- Option 1:}

One option to solve the multiobjective optimization defined by (43), (44) (or (48) , (49)) is to tune the controller in a first step, applying for example the method proposed in [33], and in a second step perform the plant design using SQP, or vice versa. The procedure presented in [33] is, in turn, another two-step iterative algorithm based on norm controllability indexes (horizon tuning + weights tuning). This iterative procedure will stop when a convergence criteria is satisfied over the objective function and optimization variables.

\section{- Option 2:}

The other studied approach is also a two-step iterative algorithm, but considering on one side the optimization of all real parameters (plant parameters, working point and controller weights) and on the other side the tuning of the MPC control horizon. In this case, for the horizon tuning, a random search based on the Solis method is used [31,37], and for the rest of real parameters, some of the multiobjective methods presented in point 2 .

If the MPC horizon is fixed in advance, Option 2 is eventually a one-step approach to solve the ID. The choice of one option or another depends on several factors, such us convergence difficulties, computing time, the need to tune the horizon $H_{c}$, etc. Although the complete problem includes all objectives and constraints for both steps, it is interesting to distribute them taking into account their influence, thus simplifying the computational load. For the plant design step, only objective $f_{1}$ is considered, together with process and controllability constraints, because it is the most relevant for that. For the controller tuning step, only objective $f_{2}$ and controllability constraints are included. When all real parameters are optimized together, both objectives must be considered together and the goal-attainment method is used to solve the problem.

\subsection{Convergence and implementation}

Although the convergence of the two steps approach is not demonstrated for a general case, it has been seen that for the activated sludge ID, only few global iterations are enough to reach a normalized converge tolerance of $10^{-3}$ in the objective function and optimization variables. The SQP and multiobjective steps convergence is based on normalized tolerances of $10^{-6}$ for the objective function, optimization variables and constraints.

As for the final algorithm implementation, the controller is based on the MPC Toolbox of MATLAB ${ }^{\circledR}$ and some modifications of Maciejowski [32]. The SQP and goal-attainment methods are implemented using the Optimization Toolbox of MATLAB ${ }^{\circledR}$, The calculations have been performed in a Quad Core Q6600 2.4 GHz CPU, with Microsoft Windows XP. 


\section{Results for case study I}

In this point some results of ID are shown for different conditions of case study I. The influence of the multiple models, the optimization methodology and the controllability indexes are addressed. The methodology followed to obtain and evaluate the results is explained here:

o First, the plant with MPC is obtained solving the ID problem using linearized models of the plant (state space models).

o Then, the plant with the controller is validated using the full nonlinear set of ASP differential equations.

Weights $W_{p}(\mathrm{~s})$ and $W_{e s}(\mathrm{~s})$ for the mixed sensitivity indexes are kept constant in order to evaluate other aspects. Note that in the ASP there are two disturbances, so weights $\mathbf{W}_{\mathbf{p}}(\mathrm{s})$ and $\mathbf{W}_{\text {esf }}(\mathrm{s})$ and sensitivity functions $\mathbf{S}(\mathrm{s}), \mathbf{M}(\mathrm{s}), \mathbf{R}_{\mathbf{d}}(\mathrm{s}), \mathbf{N}(\mathrm{s})$ are vectors and matrices with two elements (dependence in the Laplace variable $s$ for signals and transfer functions is omitted for brevity):

$\mathbf{W}_{\mathbf{p}}=\left(\begin{array}{ll}W_{p_{s i}} & W_{p_{q i}}\end{array}\right) ; \mathbf{W}_{\text {esf }}=\left(\begin{array}{ll}W_{e s f_{s i}} & W_{\text {esf } q i}\end{array}\right) ; \mathbf{S}=\left(\begin{array}{cc}\frac{s_{1}}{\tilde{s}_{i}} & 0 \\ 0 & \frac{s_{1}}{\tilde{q}_{i}}\end{array}\right) ; \mathbf{M}=\left(\begin{array}{cc}\frac{q_{r}}{s_{i}} & 0 \\ 0 & \frac{q_{r}}{q_{i}}\end{array}\right) ; \mathbf{R}_{\mathbf{d}}=\left(\begin{array}{cc}G_{d s_{i}}-K_{2} G & 0 \\ 0 & G_{d q_{i}}-K_{2} G\end{array}\right) ;$

$\mathbf{N}=\left(\begin{array}{c}\mathbf{W}_{\mathbf{p}} \cdot \mathbf{S} \cdot \mathbf{R}_{\mathbf{d}} \\ \mathbf{W}_{\text {esf }} \cdot s \cdot \mathbf{M}\end{array}\right) ; \mathbf{G}_{\mathbf{d}}=\left(\begin{array}{ll}G_{d s_{i}} & G_{d q_{i}}\end{array}\right)$

Their numerical values for case study I are the following:

$W_{p_{s i}}=\frac{7 \cdot 10^{-3} \cdot\left(\frac{s}{M_{p}}+w_{B 1}\right)}{s+w_{B 1} \cdot A_{p}} W_{p_{q i}}=\frac{0.02 \cdot\left(\frac{s}{M_{p}}+w_{B 2}\right)}{s+w_{B 2} \cdot A_{p}}$ where $w_{\mathrm{B} 1}=2500, w_{\mathrm{B} 2}=2000, A_{p}=10^{-4}, M_{p}=10^{-3}$
$W_{\text {esf } f_{s i}}=\frac{0.0175 \cdot\left(\frac{s}{M_{\text {esf }}}+w_{\mathrm{B} 3}\right)}{s+w_{B 3} \cdot A_{\text {esf }}} \quad W_{\text {esf qi }}=\frac{0.0525 \cdot\left(\frac{s}{M_{\text {esf }}}+w_{B 4}\right)}{s+w_{B 4} \cdot A_{\text {esf }}}$ where $w_{\mathrm{B} 3}=3, w_{\mathrm{B} 4}=3, A_{\text {esf }}=10^{-4}, M_{\text {esf }}=3$

The weight $\mathbf{W}_{\mathbf{p}}(\mathrm{s})$ has been chosen considering condition (24) with benchmark and real disturbance spectra, while $\mathbf{W}_{\text {eff }}(\mathrm{s})$ has been chosen to impose a certain penalty on control moves. Both weights have been determined empirically using the following procedure. First, the form of the gain has been determined, taking into account that $M_{p}\left(M_{e s f}\right)$ and $A_{p}\left(A_{e s f}\right)$ are the inverse weights gain at high and low frequencies respectively. The frequencies $w_{B 1}, w_{B 2}, w_{B 3}, w_{B 4}$ are related to the bandwidth requirement [34]. Once the form is defined, the gains are modified by an external factor in order to place them just under the inverse disturbance spectra (see Fig. 5).

The numerical bounds for the process constraints are: ret $_{d}=2.5$ hours; $r e t_{u}=8$ hours; $m l_{d}=0.001 ; m l_{u}=0.1 ; c h=0.7 \mathrm{~m} / \mathrm{h} ; \mathrm{sa}_{d}=2$ days; $\mathrm{sa}_{u}=10$ days; $\mathrm{rec}_{d}=0.05 ; \mathrm{rec}_{u}=0.9 ; \operatorname{purg}_{d}=0.03 ; \operatorname{purg}_{u}=0.3$. The dynamic constraints for MPC are: $s_{1 d}=20 \mathrm{mg} / \mathrm{l} ; \mathrm{s}_{1 u}=150$ $\mathrm{mg} / \mathrm{l} ; x_{1 d}=400 \mathrm{mg} / \mathrm{l} ; x_{1 u}=3000 \mathrm{mg} / \mathrm{l} ; q_{r d}=0 ; q_{r u}=3500 \mathrm{~m} / \mathrm{h} ; \Delta q_{r d}=0 ; \Delta q_{r u}=1000 \mathrm{~m}^{3} / \mathrm{h}$.

In order to determine the multiple models for robust ID, several criteria have been considered. The first one consists of changing the working point $\left(s_{1}\right)$, because it is very usual in the plant operation, ensuring that the designed plant satisfies the imposed conditions in a region around the nominal point (CASE 1). The two models that define the region are calculated changing the operation point $10 \mathrm{mg} / \mathrm{l}$ around the current nominal value. The second criterion to obtain the multiple models consists of modifying the influent characteristics (input flow $q_{i}$ and substrate concentration at the input $\left.s_{i}\right)\left(s_{i} \pm 100 \mathrm{mg} / \mathrm{l}, q_{i} \pm 220\right.$ $\mathrm{m}^{3} / \mathrm{h}$ ) (CASE 2). This is very interesting because the plant influent has always a large variability and so the MPC tuned is robust to those variations. Another criteria to obtain the multiple models consists of changing the plant dimensions around nominal values $\left(V_{1} \pm 300 \mathrm{~m}^{3}, A \pm 180 \mathrm{~m}^{2}\right)$, in order to give some flexibility to the designed plant in case of future redesigns or to give some error building margin (CASE 3 ).

In Table 1, Fig. 5 and Fig. 6, some results comparing performance at the worse working point of the uncertainty region for different cases are presented. A comparison with the ID case without considering multiple models in the worst working point of 
the region of CASE 2 is also shown. In the last column of the table, CASE 2 and CASE 3 for multiple models are considered together. The controllability constraint considered is $\|\mathbf{M}\|_{1}<3500$ and the control horizon is fixed to $H_{c}=10$, that is the best value obtained for this problem. For most results, the optimal tuning of the horizon has been performed in advance to reduce the computational effort, but the methodology is fully applicable for $H_{c}$ tuning if needed. The effect of local minima on the procedure has been overcome by using different starting points for each ID case. At the view of the table, all cases satisfy constraint over $\left\|\mathbf{W}_{\mathbf{p}} \cdot \mathbf{S}\right\|_{\infty}$ for disturbance rejection except the ID without multiple models, showing the advantages of performing ID with uncertainty. The cost of the ID without robustness is larger mainly because of the pumping energy; represented by the value of $q_{r 1}$ ( $q_{r 1}$ is large because no robust performance is imposed). The weight $R$ increases when more conditions are imposed to the calculation of the multiple models, but consequently, cost increases (dimensions are larger in order to satisfy performance condition throughout the region).

The influence of the optimization procedures is shown in Tables 2 and 3, where all results have been obtained considering uncertainty based on multiple models changing $s_{1}$. The ID has been performed with two different controllability constraints, $\left\|\mathbf{M}_{\mathbf{0}}\right\|_{1}<3500$ and $\left\|\mathbf{M}_{\mathbf{0}}\right\|_{1}<4000$. The results have been obtained with different starting points, choosing the best results, in order to avoid possible local minima. Note that the one-step approach (Option 2, section 7.3) optimizes all real parameters together, and the control horizon is fixed to $H_{c}=10$. In the two-step approach (Option 1, section 7.3), the number of total iterations is the sum of the iterations in each step. At the view of the results, it can be seen that the results are similar although the optimization algorithms are not equivalent (goal-attainment method for Option 2 and SQP for Option 1). This gives more reliability to the solutions obtained in the paper. Another important difference is that in the goal-attainment method (Option 2), the algorithm always gives at least one solution near to the optimum (if the rest of the problem is feasible), but when there is only one objective and the controllability is in the constraints (Option 1), the algorithm could give no solution if unfeasibility is presented, with the subsequent problems.

The comparison for different bounds over $\left\|\mathbf{M}_{\mathbf{0}}\right\|_{1}$ is obtained from comparing Tables 2 and 3 . When this bound increases (Table 3 with respect to Table 2), the control actions allowed are larger, so tuned weight $R$ is smaller.

It is also interesting to show some results of ID using different controllability indexes. In Table 4, the mixed sensitivity index in $f_{2}$ was replaced by the index (28). Both indexes are related directly to disturbance rejection, but (28) does not take into account control efforts (control increments). The results have been obtained with the one-step optimization approach ( $H_{c}$ fixed), and they are very similar, due to that, control increments and control magnitudes are linked, and the condition over $\left\|\mathbf{M}_{\mathbf{0}}\right\|_{1}$ is maintained in both cases. The only difference could be the computing time (iterations) that is usually larger for index (28). In results of Table 5 the $\|\mathbf{M}\|_{1}$ constraint is replaced by the $\|\mathbf{S}\|_{1}$, and the ID is solved for two different bounds, showing that the stricter the bound, the smaller the weight $R$ of the controller.

\section{Results for case study II}

Finally, some results of ID using the ASP model given in 6.3 with nitrogen removal are shown in Table 6, obtained by solving the optimization problem of case study II. The methodology followed is the same that for case study I.

Weights $\mathbf{W}_{\mathbf{p}}(\mathrm{s})$ and $\mathbf{W}_{\text {esf }}(\mathrm{s})$ for the mixed sensitivity indexes are also kept constant, with the following numerical values:

$$
W_{p_{S s, i n}}=\frac{40 \cdot\left(\frac{s}{2}+6\right)}{s+6 \cdot 0.3} ; W_{p_{\text {Qin }}}=\frac{70 \cdot\left(\frac{s}{1.5}+7\right)}{s+7 \cdot 0.3} ; W_{\text {esf }} \text { Ss,in }=\frac{0.0175 \cdot\left(\frac{s}{3}+4\right)}{s+4 \cdot 0.0001} ; W_{\text {esf }}=\frac{0.525 \cdot\left(\frac{s}{3}+4\right)}{s+4 \cdot 0.0001}
$$

The numerical bounds for the process constraints are: ret $_{d}=0.1$ hours; ret $_{u}=0.4$ hours; $\mathrm{ml}_{d}=0.001 ; \mathrm{ml}_{u}=0.024 ; \mathrm{ch}=9 \mathrm{~m} / \mathrm{h} ; \mathrm{sa}_{d}=2$ days; $\mathrm{sa}_{u}=10$ days; $\mathrm{rec}_{d}=0.05 ; \mathrm{rec}_{u}=0.9 ;$ purg $_{d}=0.03 ;$ purg $_{u}=0.3 ; S_{N O, d n i t, d}=3 \mathrm{mg} / \mathrm{l} ; S_{\mathrm{NO}, \mathrm{dnit}, u}=10 \mathrm{mg} / \mathrm{l} ; Q_{r 1 d}=0 ; Q_{r 1 u}=50000$ $\mathrm{m}^{3} / \mathrm{h} ; \Delta \mathrm{Q}_{\mathrm{r} 1 d}=0 ; \Delta Q_{\mathrm{r} 1 \mathrm{u}}=1000 \mathrm{~m}^{3} / \mathrm{h}$. 
The ID has been performed with two different controllability constraints, $\left\|\mathbf{M}_{0}\right\|_{1}<2400$ and $\left\|\mathbf{M}_{0}\right\|_{1}<2570$. The Option 1 approach for solving the optimization has been considered (see 7.2), and $H_{c}=10$ is fixed in advance. The results are analogous to case study $I$, because when the bound over $\left\|\mathbf{M}_{\mathbf{0}}\right\|_{1}$ is stricter, the disturbance rejection is worse because the control magnitudes are limited (Fig. 7), and the cost increases a bit to satisfy the requirements of disturbance rejection. In order to perform a further validation of the results, a comparison of performance using an MPC without terminal penalty and not obtained within the ID framework is presented. For that controller, the plant is fixed, with the parameters of the first column of Table 6. The MPC tuning parameters are arbitrarily selected to $R=0.0020$ and $H_{c}=10$. At the view of the $S_{N O \text {,dnit }}$ deviations from

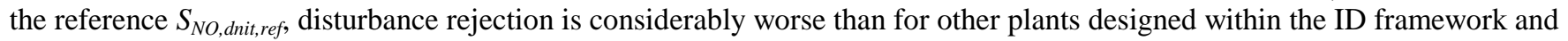
a stable MPC.

\section{Conclusions}

In this work an Integrated Design (ID) procedure to obtain optimal plants and control systems for activated sludge processes with stable MPC has been proposed. The MPC is an infinite horizon controller, implemented as a penalty term to guarantee stability. The problem is based on optimization including investment, operating costs, and dynamical indexes based on the weighted sum of the $\mathrm{H}_{\infty}$ and $l_{1}$ norms of different closed loop transfer functions matrices of the system, following a multiobjective methodology. Some robustness conditions are also included as constraints to guarantee that the resulting plant and control system designs are robust in the face of nonlinearities and disturbances acting on the process. The quality of the plants obtained with this ID procedure is similar to those obtained using ISE, together with other dynamical indexes, but the computational time is much larger in the last case.

Some comparisons show the effectiveness of the new approach, where the optimal plant and MPC obtained produce better disturbance rejection than those obtained following a classical design. The methodology proposed for ID is general and it has been extended to nitrogen control changing only the nonlinear process equations and their linearization appropriately. Additionally, this methodology can be used for ID of chemical and biotechnological processes. Although the models considered may be too simple to simulate, such a complex process, we do not intend to provide a precise simulation here. For classical design, steady state processes are usually considered, and in this work we improve the design procedure by using dynamical models. The use of more complex models for ID would be impractical, and if needed, a full simulator could be used after the application of the methodology proposed.

\section{Acknowledgments}

The authors gratefully acknowledge the support of the Spanish Government through the MICINN project DPI2009-14410-C0201 .

\section{References}

[1] M. L. Luyben, Analyzing the Interaction Between Process Design and Process Control. Ph.D. Thesis, Princeton University, 1993.

[2] M. L. Luyben, C. Floudas. Analyzing the interaction of design and control-1. A multiobjective framework and application to binary distillation synthesis. Comp. Chem. Eng. Vol. 18, nº 10 (1994) 933-969.

[3] C. Schweiger, C. Floudas. Interaction of design and control: Optimization with dynamic models. In W. Hager, P. Pardalos (Eds.). Optimal Control: Theory, Algorithms and Applications. Kluwer, 1997, pp. 388-435.

[4] I. Kookos, J. Perkins. An algorithm for simultaneous process design and control. Ind. Eng. Chem. Res. 40 (2001), 40794088.

[5] V. Sakizlis, J. D. Perkins, E. N. Pistikopoulos. Recent advances in optimization-based simultaneous process and control design. Computers and Chemical Engineering, 28, (2004) 2069-2086.

[6] P. Seferlis. C. Georgiadis (Eds.). The Integration of Process Design and Control. Computer-Aided Chemical Engineering, 17. Elsevier (2004)

[7] R. Grosch, M. Mönnigmann, W. Marquardt. Integrated design and control for robust performance: Application to an MSMPR crystallizer. Journal of Process Control, 18 (2008) 173-188.

[8] A. Flores-Tlacuahuac, L. T. Biegler. Integrated Control and Process Design During Optimal Polymer Grade Transitions Operations. Comp. Chem. Eng., Vol. 32, nº11 (2008) 2823-2837.

[9] P. Vega, G. Gutiérrez. Optimal Design Control and Operation of wastewater treatment plants. European Control Conference (Germany), (1999) 
[10] M. Francisco, P. Vega. Diseño Integrado de procesos de depuración de aguas utilizando Control Predictivo Basado en Modelos. Rev. Iberoamericana de Automática e Informática Industrial, Vol. 3, nº 4 (2006) 88-98.

[11] R. Baker, C.L.E. Swartz. Interior Point Solution of Integrated Plant and Control Design Problems with Embedded MPC. AIChE Annual Meeting (San Francisco), (2006).

[12] S. Sakizlis, J. D. Perkins, E. N. Pistikopoulos, Parametric controllers in simultaneous process and control design optimization. Ind. Eng. Chem. Res., 42, (2003) 4545-4563.

[13] M. Miranda, J. M. Reneaume, X. Meyer, M. Meyer, F. Szigeti. Integrating process design and control: An application of optimal control to chemical processes. Chemical Engineering and Processing, 47. (2008) 2004-2018.

[14] F. Nejjari, J. Quevedo. Predictive Control of a Nutrient Removal Biological plant. American Control Conference, (Boston, Massachusetts) (2004).

[15] M. Francisco, P. Vega, O. Pérez, M. Poch. Dynamic Optimization for Activated Sludge Integrated Design. European Control Conference (Cambridge, UK), (2003).

[16] G. Gutierrez, P. Vega. Integrated Design of Biological Processes and their Control System including Closed Loop Properties for Disturbances Rejection. IFAC World Congress (Barcelona, Spain) (2002).

[17] A. Rivas, E. Ayesa, A. Galarza, A. Salterain. Application of mathematical tools to improve the design and operation of activated sludge plants. Case study: the new WWTP of Galindo-Bilbao. Part I: Optimum design. Water Science and Technology. Vol. 43, no 7 (2001) 157-165.

[18] A. Galarza, E. Ayesa, M. T. Linaza, A. Rivas, A. Salterain. Application of mathematical tools to improve the design and operation of activated sludge plants. Case study: the new WWTP of Galindo-Bilbao. Part II: Operational strategies and automatic controllers. Water Science and Technology. Vol. 43, nº 7 (2001) 167-174.

[19] M. Francisco, P. Vega. Integrated Design of Wastewater Treatment Processes using Model Predictive Control. European Control Conference (Kos, Greece), (2007) 5333-5340.

[20] O. A. Z. Sotomayor, C. García. Model-Based Predictive Control of a pre-denitrification plant: a linear state-space model approach. IFAC World Congress, (Barcelona,Spain) (2002).

[21] J. B. Rawlings, K. R. Muske, The stability of constrained receding horizon control. IEEE transactions on automatic control, 38, nº 10 (1993) 1512-1516.

[22] P. O. M. Scokaert, J. B. Rawlings. Constrained Linear Quadratic Regulation. IEEE Trans. on Automatic Control, vol. 43, $\mathrm{n}^{\mathrm{o}} 8$ (1998) 1163-1169.

[23] M. Francisco, P. Vega. Norm based approaches for Integrated Design of Wastewater Treatment Plants. Proceedings of ECCE-6 (Copenhague, Denmark) (2007).

[24] M. Morari, E. Zafiriou, Robust process control. Prentice Hall. (1989).

[25] F. Tadeo, T. Holohan, P. Vega. $l_{1}$-optimal regulation of a pH control plant. Computers and Chemical Engineering, Vol. 22, Suppl., (1998) S459-S466.

[26] M. Francisco, P. Vega. Multi-model approaches for Integrated Design of Wastewater Treatment Plants with Model Predictive Control. 17 $7^{\text {th }}$ IFAC World Congress. (Seoul, Korea) (2008)

[27] V. Sakizlis, J. D. Perkins, E. N. Pistikopoulos, Simultaneous process and control design using mixed integer dynamic optimization and parametric programming. In P. Seferlis, M.C. Georgiadis (Eds.). The Integration of Process Design and Control. Computer-Aided Chemical Engineering, 17. Elsevier (2004), pp. 187-215

[28] O. H. Sendín, C. G. Moles, A. A. Alonso, J. R. Banga. Multi-objective Integrated Design and Control using Stochastic Global Optimization Methods. In P. Seferlis, M.C. Georgiadis (Eds.). The Integration of Process Design and Control. Computer-Aided Chemical Engineering, 17. Elsevier (2004), pp. 555-581.

[29] S. Revollar, R. Lamanna, P Vega. Algorithmic synthesis and integrated design for activated sludge processes using genetic algorithms. ESCAPE.(Barcelona, Spain) (2005)

[30] K. M. Miettinen. Nonlinear Multiobjective Optimization. Ed. Kluwer (1999).

[31] K. Deb. Multi-Objective Optimization using Evolutionary Algorithms. Ed. Wiley (2002).

[32] J. M. Maciejowsky. Predictive Control with Constraints. Prentice Hall, (2002).

[33] M. Francisco, P. Vega. Automatic tuning of Model Predictive Controllers based on Multiobjective Optimization. Latin American Applied Research (2010, in press)

[34] S. Skogestad, I. Postlethwaite. Multivariable Feedback Control, Analysis and Design. John Wiley \& Sons, (1997).

[35] R. Moreno, C. De Prada, J. Lafuente, M. Poch, G. Montague. Non-linear predictive control of dissolved oxygen in the activated sludge process. IFAC BIO 2, Ed. Pergamon Press, (1992) pp. 289-298.

[36] J. B. Copp. The COST Simulation Benchmark: Description and Simulator Manual. Office for Official Publications of the European Community. ISBN 92-894-1658-0. (2002)

[37] F. J. Solis, R. J-B. Wets. Minimization by random search techniques. Mathematics of Operations Research, Vol. 6, (1981) 19-30. 


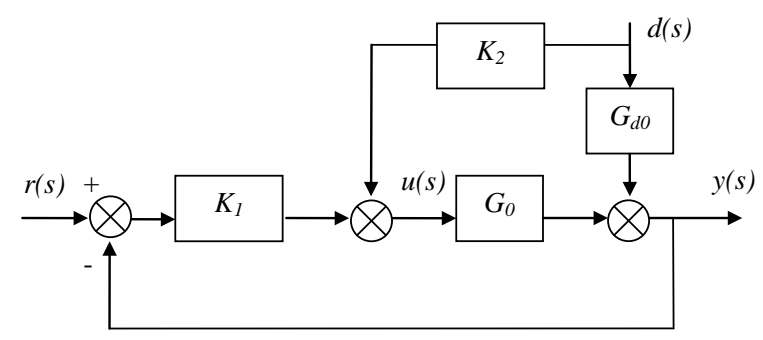

Fig. 1: Nominal closed loop system

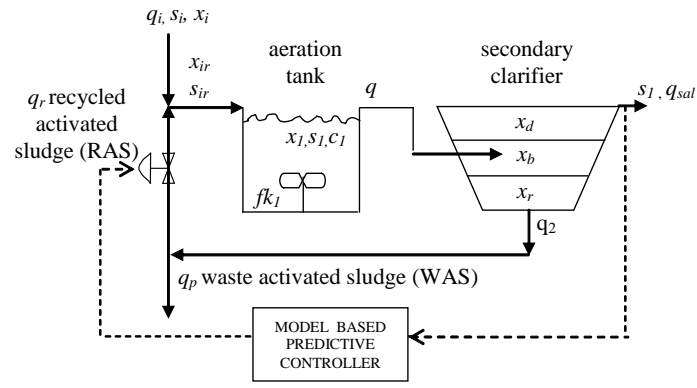

Fig. 2. Plant and controller layout for the ASP for substrate elimination
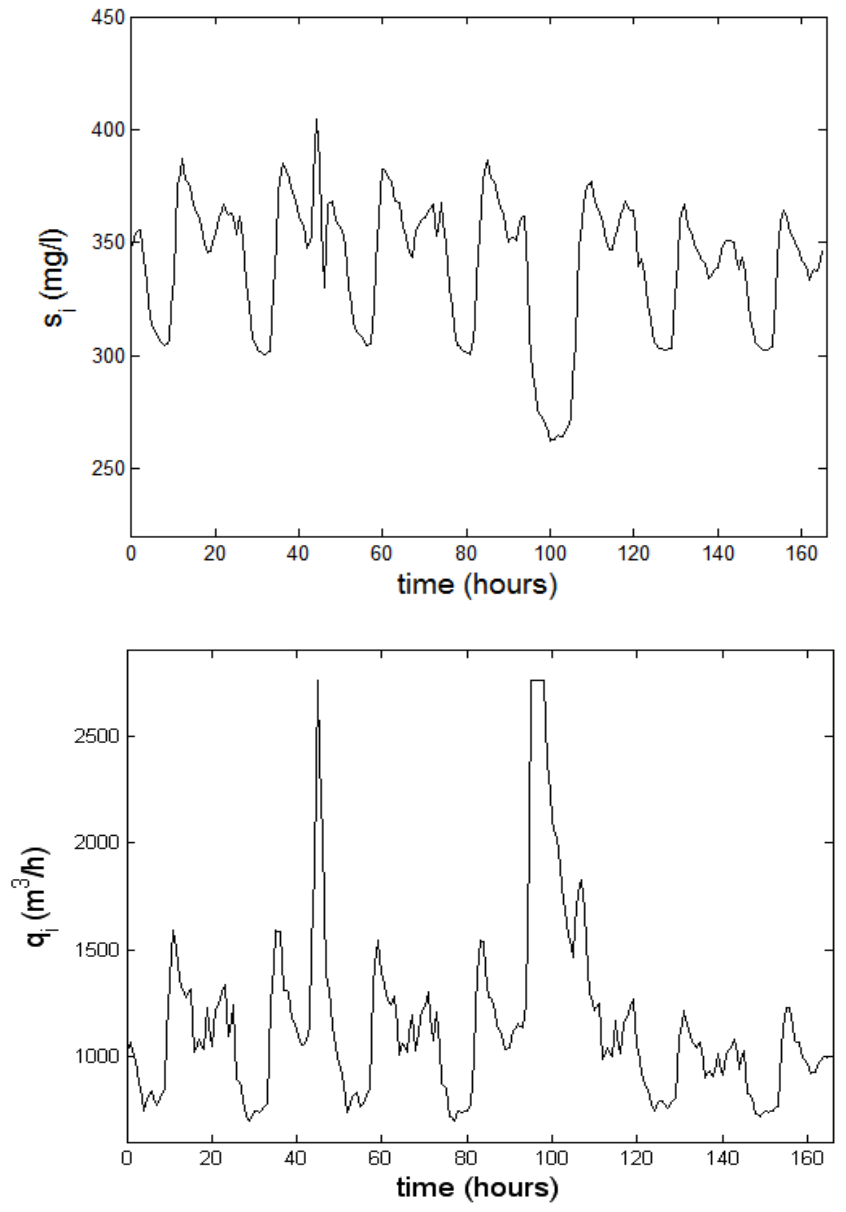

Fig. 3: Storm weather disturbances at the influent $\left(s_{i}, q_{i}\right)$ 


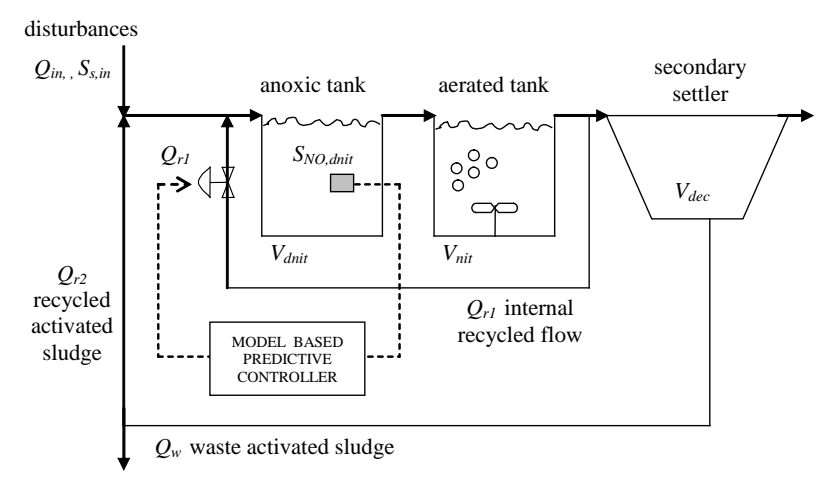

Fig. 4. Plant and controller layout for the ASP for nitrogen removal
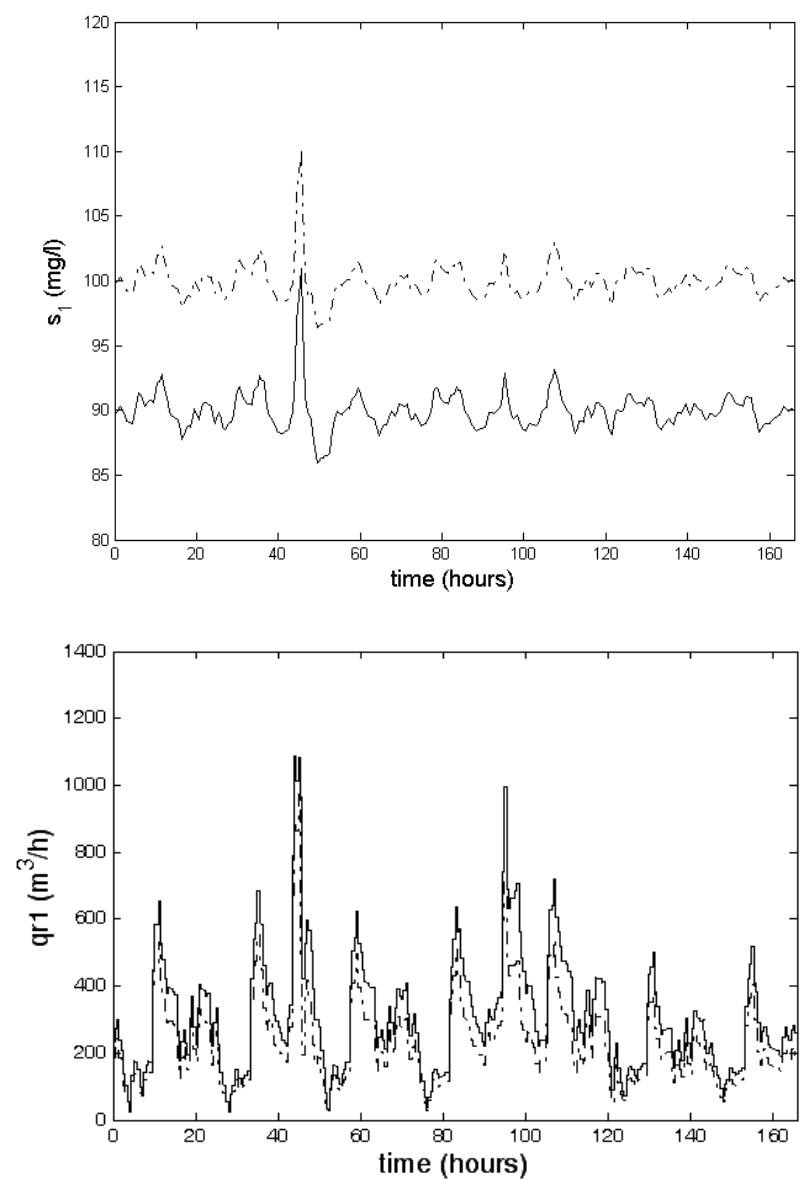

Fig. 5: A comparison of $s_{1}$ and $q_{r}$ for the plant designed with robust ID (uncertainty of CASE 1), working in the nominal point (dash dotted line) and working in the limit of the region (solid line) 


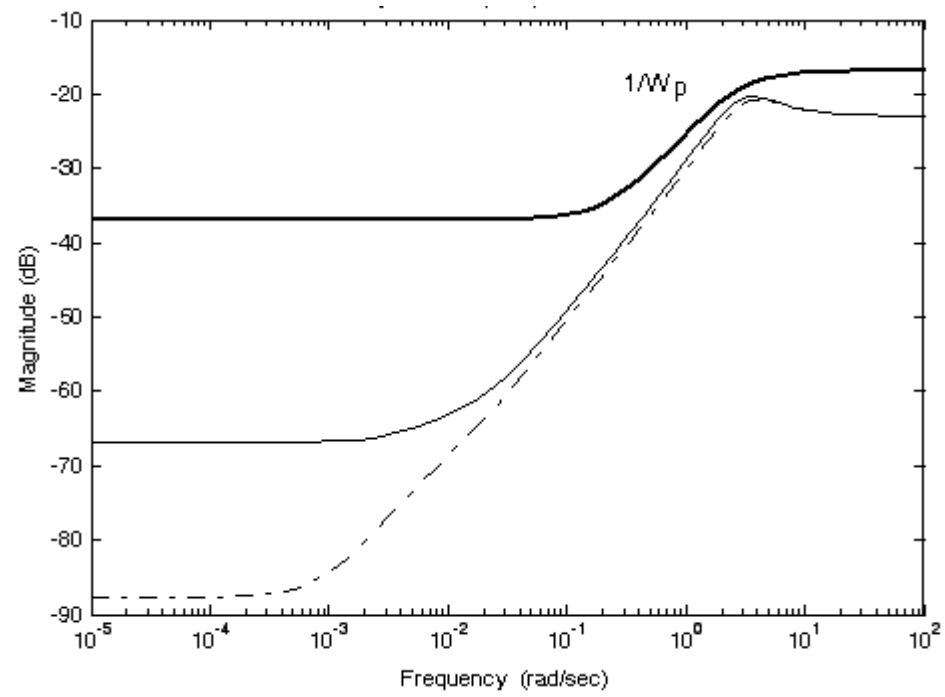

Fig. 6: Output sensitivity functions for the closed loop plant designed with robust ID with CASE 1 of multiple models, working in the limit of the region (solid line) and in the nominal point (dash dotted line), together with magnitude of weight $W_{p_{s i}}{ }^{-1}$ (solid thick line)
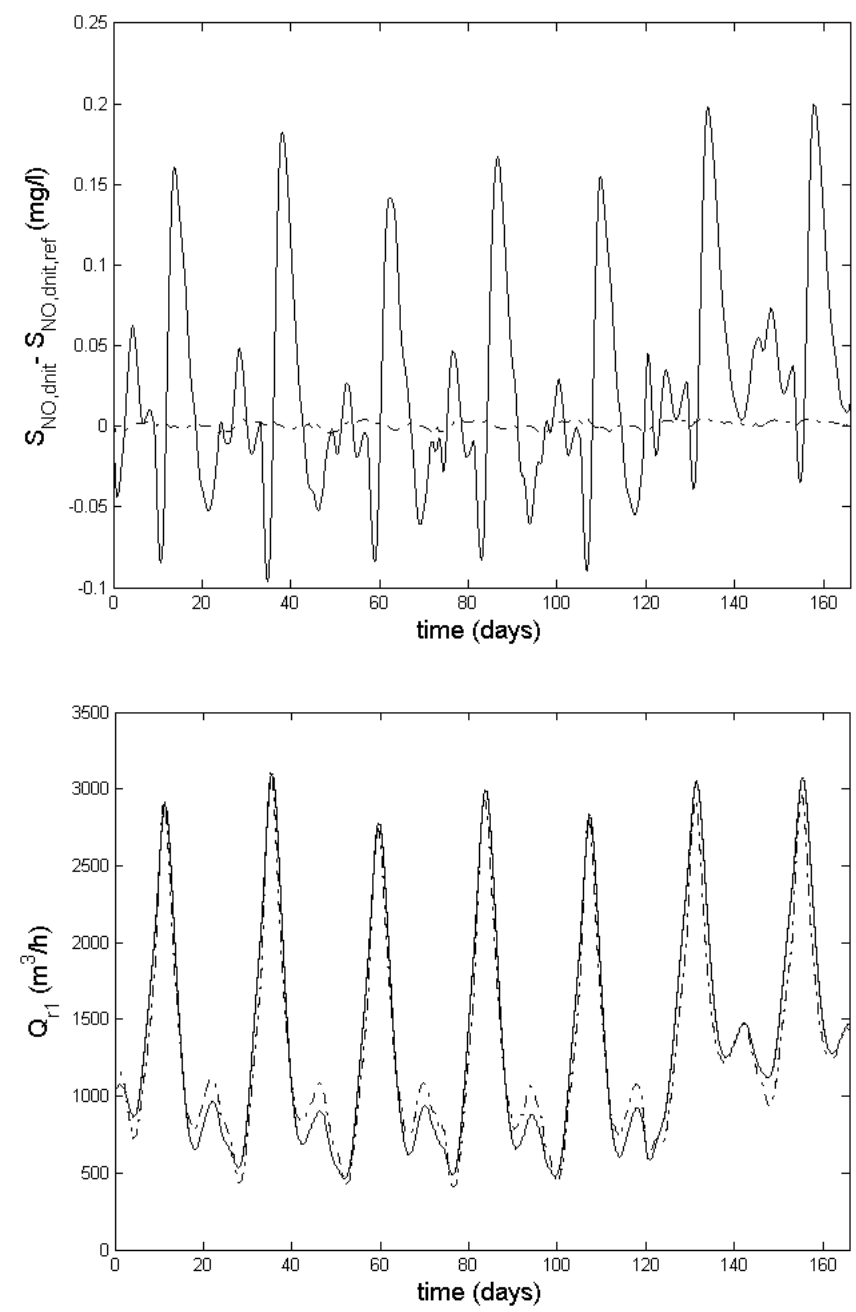

Fig. 7: A comparison of $S_{N O, d n i t}-S_{N O, d n i t, r e f}$ and $Q_{r 1}$ for the plants designed with controllability constraint $\left\|\mathbf{M}_{\mathbf{0}}\right\|_{1}<2570$ (dash dotted line) and $\left\|\mathbf{M}_{0}\right\|_{1}<2400$ (solid line). 
Table 1

Results for different robust ID cases when working in the worse point of the uncertainty region

\begin{tabular}{|c|c|c|c|c|}
\hline & $\begin{array}{l}\text { ID with } \\
\text { single } \\
\text { models }\end{array}$ & CASE 1 & CASE 2 & $\begin{array}{l}\text { CASE } 2 \\
\text { CASE } 3\end{array}$ \\
\hline$R$ & 0.00699 & 0.00737 & 0.00647 & 0.00589 \\
\hline$V_{1}\left(\mathrm{~m}^{3}\right)$ & 3605.5 & 3628 & 3923 & 4443 \\
\hline$A\left(\mathrm{~m}^{2}\right)$ & 2452 & 2449.4 & 2445.5 & 2225.5 \\
\hline$s_{1}(\mathrm{mg} / \mathrm{l})$ & 100.0 & 90.0 & 99.0 & 95.0 \\
\hline$q_{r}\left(\mathrm{~m}^{3} / \mathrm{h}\right)$ & 570.5 & 257.5 & 354.6 & 362.3 \\
\hline$s_{i}(\mathrm{mg} / \mathrm{l})$ & 400 & 340 & 400 & 400 \\
\hline$q_{i}\left(\mathrm{~m}^{3} / \mathrm{h}\right)$ & 1280 & 1150 & 1280 & 1280 \\
\hline$\left\|\mathbf{W}_{\mathbf{p}} \cdot \mathbf{S} \cdot \mathbf{R}_{\mathrm{d}}\right\|_{\infty}$ & 33.829 & 1 & 1 & 1 \\
\hline$\|\mathbf{M}\|_{1}$ & 4889.5 & 3632.4 & 4889.5 & 5558.3 \\
\hline Cost & 0.999 & 0.184 & 0.364 & 0.471 \\
\hline $\begin{array}{l}\text { max. deviation } \\
\text { from } s_{1}(\mathrm{mg} / \mathrm{l})\end{array}$ & $\begin{array}{l}\text { MPC non } \\
\text { feasible }\end{array}$ & 17.9 & 26.3 & 23.7 \\
\hline
\end{tabular}

Table 2

Results for ID with different optimization methods (I)

\begin{tabular}{lll}
\hline$\left\|\mathbf{M}_{\mathbf{0}}\right\|_{1}<3500$ & $\begin{array}{l}\text { One step } \\
\text { optimization }\end{array}$ & $\begin{array}{l}\text { Two iterative } \\
\text { steps optimization }\end{array}$ \\
\hline$R$ & 0.00737 & 0.00694 \\
$V_{1}\left(\mathrm{~m}^{3}\right)$ & 3628 & 3616.5 \\
$A\left(\mathrm{~m}^{2}\right)$ & 2449.4 & 2459.4 \\
$\mathrm{~s}_{1}(\mathrm{mg} / \mathrm{l})$ & 100.0 & 100.0 \\
max. deviation & 13.98 & 13.88 \\
from $\mathrm{s}_{1}(\mathrm{mg} / \mathrm{l})$ & & \\
$\left\|\mathbf{w}_{\mathbf{p}} \cdot \mathbf{S}_{\mathbf{0}} \cdot \mathbf{R}_{\mathrm{d} 0}\right\|_{\infty}$ & 0.909 & 0.879 \\
$\left\|\mathbf{M}_{\mathbf{0}}\right\|_{1}$ & 3403.3 & 3500 \\
Cost & 0.1420 & 0.1428 \\
Computing time (s) & 500.18 & 271.31 \\
Global Iterations & 1 & 4 \\
Total Iterations & 76 & 57 \\
\hline
\end{tabular}

Table 3

Results for ID with different optimization methods (II)

\begin{tabular}{lll}
\hline$\left\|\mathbf{M}_{\mathbf{0}}\right\|_{1}<4000$ & $\begin{array}{l}\text { One step } \\
\text { optimization }\end{array}$ & $\begin{array}{l}\text { Two iterative } \\
\text { steps optimization }\end{array}$ \\
\hline$R$ & 0.00665 & 0.00575 \\
$V_{1}\left(\mathrm{~m}^{3}\right)$ & 3796.3 & 3604.3 \\
$A\left(\mathrm{~m}^{2}\right)$ & 2308.9 & 2452 \\
$s_{1}(\mathrm{mg} / \mathrm{l})$ & 100.0 & 100.0 \\
max. deviation & 13.66 & 13.68 \\
from $s_{1}(\mathrm{mg} / \mathrm{l})$ & & \\
$\left\|\mathbf{w}_{\mathbf{p}} \cdot \mathbf{S}_{\mathbf{0}} \cdot \mathbf{R}_{\mathrm{d} 0}\right\|_{\infty}$ & 0.849 & 0.789 \\
$\left\|\mathbf{M}_{\mathbf{0}}\right\|_{1}$ & 3532.5 & 3808.4 \\
Cost & 0.14395 & 0.14194 \\
Computing time (s) & 518.74 & 566.79 \\
Global Iterations & 1 & 4 \\
Total Iterations & 75 & 117 \\
\hline
\end{tabular}


Table 4

Results for ID with different controllability indexes

\begin{tabular}{lll}
\hline & $f_{2}=\left\|\mathbf{N}_{\mathbf{0}}\right\|_{\infty}$ & $f_{2}=\left\|G_{0} \mathbf{K}_{2}+\mathbf{G}_{\mathbf{d} \mathbf{0}}\right\|_{1}$ \\
\hline$R$ & 0.00696 & 0.00724 \\
$V_{1}\left(\mathrm{~m}^{3}\right)$ & 3632.1 & 3605.4 \\
$A\left(\mathrm{~m}^{2}\right)$ & 2432 & 2452.1 \\
$s_{1}(\mathrm{mg} / \mathrm{l})$ & 100.0 & 100.0 \\
max. deviation & 13.97 & 14.09 \\
from $s_{1}(\mathrm{mg} / \mathrm{l})$ & & \\
$\left\|\mathbf{W}_{\mathbf{p}} \cdot \mathbf{S}_{\mathbf{0}} \cdot \mathbf{R}_{\mathrm{d} \mathbf{0}}\right\|_{\infty}$ & 0.881 & 0.903 \\
$\left\|G_{0} \mathbf{K}_{\mathbf{2}}+\mathbf{G}_{\mathbf{d} 0}\right\|_{1}$ & 119.7 & 119.6 \\
$\left\|\mathbf{N}_{\mathbf{0}}\right\|_{\infty}$ & 0.954 & 0.974 \\
$\left\|\mathbf{M}_{\mathbf{0}}\right\|_{1}$ & 3498.6 & 3443.4 \\
Cost & 0.14198 & 0.14194 \\
Global Iterations & 1 & 1 \\
Total Iterations & 35 & 71 \\
\hline
\end{tabular}

Table 5

Results for ID with different controllability constraints

\begin{tabular}{lll}
\hline & $\left\|\mathbf{S}_{\mathbf{0}}\right\|_{1}<12$ & $\left\|\mathbf{S}_{\mathbf{0}}\right\|_{1}<15$ \\
\hline$R$ & 0.00143 & 0.00162 \\
$V_{1}\left(\mathrm{~m}^{3}\right)$ & 3605.3 & 3605.7 \\
$A\left(\mathrm{~m}^{2}\right)$ & 2451.9 & 2451.8 \\
$\mathrm{~s}_{1}(\mathrm{mg} / \mathrm{l})$ & 100.0 & 100.0 \\
max. deviation & 12.37 & 12.44 \\
from $\mathrm{s}_{1}(\mathrm{mg} / \mathrm{l})$ & & \\
$\left\|\mathbf{w}_{\mathbf{p}} \cdot \mathbf{S}_{\mathbf{0}} \cdot \mathbf{R}_{\mathrm{d} 0}\right\|_{\infty}$ & 0.460 & 0.475 \\
$\left\|\mathbf{M}_{\mathbf{0}}\right\|_{1}$ & 6986.5 & 6449.4 \\
$\left\|\mathbf{S}_{\mathbf{0}}\right\|_{1}$ & 12.00 & 12.57 \\
Cost & 0.14194 & 0.14194 \\
Global Iterations & 4 & 4 \\
Total Iterations & 103 & 194 \\
\hline
\end{tabular}

Table 6

Results for ID with different controllability constraints and the process for nitrogen removal compared with MPC controller without terminal penalty

\begin{tabular}{llll}
\hline & $\left\|\mathbf{M}_{\mathbf{0}}\right\|_{1}<2400$ & $\left\|\mathbf{M}_{\mathbf{0}}\right\|_{1}<2570$ & $\begin{array}{c}\text { MPC without } \\
\text { terminal penalty }\end{array}$ \\
\hline$R$ & 0.00373 & 0.00001 & 0.0020 \\
$V_{\text {dnit }}\left(\mathrm{m}^{3}\right)$ & 7944.3 & 7361.5 & 7944.3 \\
$V_{\text {nit }}\left(\mathrm{m}^{3}\right)$ & 8062.2 & 6878.9 & 8062.2 \\
$V_{\text {dec }}\left(\mathrm{m}^{3}\right)$ & 2587.6 & 2505.8 & 2587.6 \\
$S_{N O, \text { dnit }}(\mathrm{mg} / \mathrm{l})$ & 8.1707 & 8.33 & 8.1707 \\
$\max \left(S_{N O, d n i t}-S_{N O, d n i t, r e f}\right)(\mathrm{mg} / \mathrm{l})$ & 0.1999 & 0.0045 & 0.4302 \\
$\left\|\mathbf{w}_{\mathbf{p}} \cdot \mathbf{S}_{\mathbf{0}} \cdot \mathbf{R}_{\mathrm{d} 0}\right\|_{\infty}$ & 0.95411 & 0.0410 & 1.7429 \\
$\left\|\mathbf{M}_{\mathbf{0}}\right\|_{1}$ & 2400 & 2570 & 1799.4 \\
$\left\|\mathbf{S}_{\mathbf{0}}\right\|_{1}$ & 0.523 & 0.0077 & 0.7827 \\
Cost & 0.05100 & 0.0401 & 0.0510 \\
Global Iterations & 3 & 3 & - \\
Total Iterations & 63 & 66 & - \\
\hline
\end{tabular}

